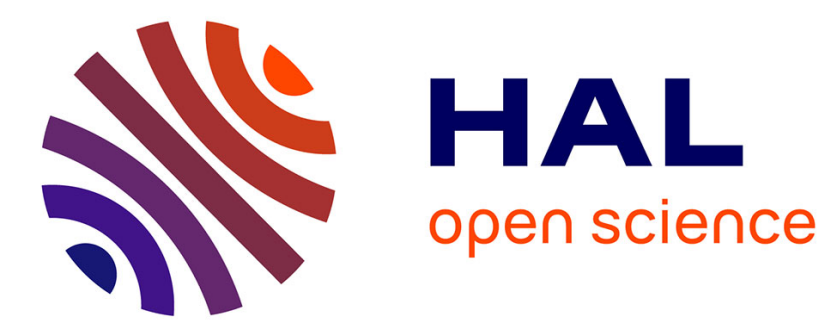

\title{
Stationary shock-like transition fronts in dispersive systems
}

Sergey Gavrilyuk, Boniface Nkonga, Keh-Ming Shyue, Lev Truskinovsky

\section{To cite this version:}

Sergey Gavrilyuk, Boniface Nkonga, Keh-Ming Shyue, Lev Truskinovsky. Stationary shock-like transition fronts in dispersive systems. 2020. hal-01958328v2

\section{HAL Id: hal-01958328 \\ https://hal.science/hal-01958328v2}

Preprint submitted on 1 Sep 2020

HAL is a multi-disciplinary open access archive for the deposit and dissemination of scientific research documents, whether they are published or not. The documents may come from teaching and research institutions in France or abroad, or from public or private research centers.
L'archive ouverte pluridisciplinaire HAL, est destinée au dépôt et à la diffusion de documents scientifiques de niveau recherche, publiés ou non, émanant des établissements d'enseignement et de recherche français ou étrangers, des laboratoires publics ou privés. 


\title{
Stationary shock-like transition fronts in dispersive systems
}

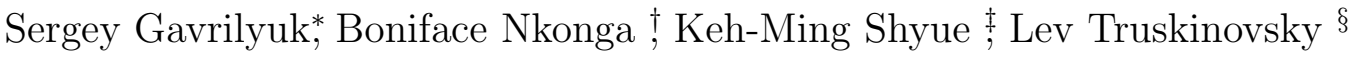

July 24, 2020

\begin{abstract}
We show that, contrary to popular belief, lower order dispersive regularization of hyperbolic systems does not exclude the development of the localized shock-like transition fronts. To guide the numerical search of such solutions, we generalize Rankine-Hugoniot relations to cover the case of higher order dispersive discontinuities and study their properties in an idealized case of a transition between two periodic wave trains with different wave lengths. We present evidence that smoothed stationary fronts of this type are numerically stable in the case when regularization is temporal and one of the adjacent states is homogeneous. In the zero dispersion limit such shock-like transition fronts, that are not traveling waves and apparently require for their description more complex anzats, evolve into traveling wave type jump discontinuities.
\end{abstract}

\section{Introduction}

Hyperbolic systems, representing conservation laws and describing non-dissipative wave-like processes, are known to generate discontinuities even under smooth initial data $[6,7]$. Dissipative regularization can resolve such singularities replacing them with smooth transition fronts [66, 55, 52]. The situation is more complex in the case of dispersive regularization which leaves the system dissipation-free remaining faithful to the conservative nature of the original problem [66, 32, 33].

Dispersive regularization is usually either spatial or temporal. Spatial regularization brings finite length scales and is usually accomplished through higher space derivatives or by space discretization $[65,62,14]$. Steady discontinuities are then replaced by non-steady modulated wave trains $[26,30]$. Instead, temporal regularization, bringing finite time scales, either through memory kernels or through higher order time derivatives $[49,45,18,20,8,4]$, was shown to support at least stationary localized shocks which survive as smoothed transients in a class of shallow water models, including unidirectional (Benjamin-Bona-Mahony) [15] and bidirectional (Boussinesq) [16] equations. Localized traveling wave (TW) solutions were also found in models with high-order dispersion where they correspond to heteroclinics connecting periodic orbits $[1,5,13,56,31]$.

In this paper we provide the first evidence that even the low-order dispersive temporal regularization can support stationary shock-like transition fronts that are not dispersive TW. As a prototypical example we use the simplest strictly hyperbolic $p$-system $[6,52]$ which is regularized

\footnotetext{
*Aix Marseille Univ, CNRS, IUSTI, UMR 7343, Marseille, France, sergey.gavrilyuk@univ-amu.fr

†Université Côte d'Azur \& Inria Sophia-Antipolis Méditerranée, CNRS LJAD, Nice, France, Boniface.Nkonga@unice.fr

¥Institute of Applied Mathematical Sciences, National Taiwan University, Taipei 106, Taiwan, shyue@ntu.edu.tw

§PMMH, CNRS UMR 7636, ESPCI PSL, 10 Rue Vauquelin, 75005, Paris, France, lev.truskinovsky@espci.fr
} 
by the lowest time derivatives as it is done, for instance, in the theory of bubbly fluids [3]. To guide the numerical search for localized transition fronts, we first use the Hamilton principle to derive a set of higher order Rankine-Hugoniot (RH) jump conditions for a general dispersively regularized model. We use the ensuing kinematic and dynamic compatibility conditions to identify admissible dispersive discontinuities in the general case and then work out in full detail the case when the transition is between two periodic TWs with different wave lengths. While such composite waves, even if they are RH admissible, are not smooth TW solutions by themselves, they play an important role as building blocks for the construction of the desired shock-like transition fronts.

We ultimately limit our construction to the special case when the transition fronts are stationary and one of the coexisting states is homogeneous. We show that if our generalized RH conditions are satisfied, the obtained composite solutions can withstand the spreading effect of dispersion. In such solutions the 'destabilizing' activity of the internal time scale is effectively disabled by the stationarity of the front, which is similar to what observed in $[15,16]$.

To illustrate the numerical stability of the constructed transition fronts, we conduct numerical experiments using as a convenient example a fully nonlinear Serre-Green-Naghdi (SGN) system designed to describe long gravity waves [51, 59, 23, 24]. Our tests cover a broad class of Riemanntype initial value problems involving 'cold' (homogeneous) states arbitrarily narrowly linked to 'hot' (oscillatory) states. We show that in a certain parameter range, numerical evolution of such 'composite' initial data leads to the formation of an apparently stationary, shock-like transition fronts which satisfy our generalized RH jump conditions.

In a first approximation, the emerging transition fronts can be described as truncated solitary waves smoothly joining the matching cnoidal-type waves. More precisely, one far field state is a degenerate periodic solution with zero wave number and another one is a co-propagating wave with a finite wave number. The two waves are connected by a non-analytic transition region occurring over the length scale of a single oscillation of a periodic wave. At the time scale of numerical simulations the size of the transition region remained unchanged, which clearly distinguishes the obtained solutions from conventional dispersive shocks and transient dispersive discontinuities. We also stress that such shock-like fronts emerge robustly from a whole class of initial data.

The fact that numerically resolved transition fronts sharpen as the dispersive time scale tends to zero while the limiting periodic patterns maintain their amplitude suggest weak convergence. However, similar to the examples presented in $[15,16]$, the formal weak limits of the obtained fronts are unstable (entropically non-admissible) in the framework of the original $p$-system. They owe their stability exclusively to dispersive regularization and therefore, instead of the $p$-system, the limiting measure valued profiles $[37,38,9]$ can be expected to serve as admissible weak solutions of the Whitham-type higher order hyperbolic system [66, 12, 14, 57]. It can be either a system of conservation laws for the parameters of modulated periodic traveling waves or a higher order system of multiphase modulation equations.

The rest of the paper is organized as follows. In Section 2 we use the action principle to derive the generalized jump conditions for a regularized model with spatial and temporal dispersion. In Section 3 we focus on the case of temporal dispersion, introduce periodic TW solutions and study the possibility of discontinuous and smoothed connections between them. A specific example (SGN model) is considered in Section 4. Numerical evidence of dynamical stability for the constructed stationary shocks-like transformation fronts is presented in Section 5. To show robustness of the obtained picture we reproduce it for a simplified Boussinesq-type model with linearized dispersion. The final Section 6 contains our conclusions. The numerical algorithm and some other technical issues are discussed in four Appendices. 


\section{Regularized model}

Variational formalism. Conservative models in continuum mechanics can be obtained from the Hamilton principle which requires that the action functional is stationary on the trajectories of the system [22]. For our purposes it will be sufficient to consider the functionals of the form

$$
\mathcal{L}=\int_{\Omega} L\left(x_{, a}, x_{, a b}\right) d q^{1} d q^{2},
$$

where $L$ is a Lagrangian density. The deformation is defined by the function $x\left(q^{a}\right), a=1,2$, representing trajectories of material particles, where $q^{1}=q$ is the spatial Lagrangian coordinate and $q^{2}=t$ denotes time. The subscript after a comma in (1) indicates partial derivative. The integration in (1) is over the two-dimensional space-time domain $\Omega$ representing the evolving body between the time instants $t=t_{0}$ and $t=t_{1}$. In such representation of dynamics the trivial identities $x_{, a b}=x_{, b a}$ can be viewed as describing mass balance.

We first obtain the Euler-Lagrange equations

$$
\left(\frac{\delta L}{\delta x_{, a}}\right)_{, a}=0
$$

where $\frac{\delta L}{\delta x_{, a}}=\frac{\partial L}{\partial x_{, a}}-\left(\frac{\partial L}{\partial x_{, a d}}\right)_{, d}$ is the variational derivative and the summation over repeated indexes is implied. Due to the symmetry of the second derivatives one has to count the mixed derivatives in (2) only once, so the summation is over $a$ and $d \geq a$. The equilibrium condition (2) usually represent the linear momentum balance.

In view of (2), and the translational invariance of the Lagrangian density, one can also obtain from (1) two conservation laws (Noether identities)

$$
\left(x_{, b} \frac{\delta L}{\delta x_{, a}}+x_{, c b} \frac{\partial L}{\partial x_{, c a}}-L \delta_{b}^{a}\right)_{, a}=0
$$

where $\delta_{b}^{a}$ is the Kronecker symbol. The relations (3) must be satisfied along the actual trajectory of the system. The temporal component of (3) is the energy balance equation; the spatial component is known in fluid mechanics as the Bernoulli equation [2] and in solid mechanics as the Eshelby equation [44].

Suppose now that the domain $\Omega$ contains a propagating interface $\Sigma$. Suppose also that the particle trajectories are continuous on $\Sigma$ so $[x]=0$ but their derivatives experience discontinuities; here $[f]=f^{+}-f^{-}$with the superscripts \pm denoting the limiting values of $f$. On such interfaces (if they exist) the action principle imposes the constraints which can be viewed as the generalized $\mathrm{RH}$ jump conditions

$$
\begin{gathered}
{\left[\frac{\delta L}{\delta x_{, a}}\right] n_{a}=0,} \\
{\left[\frac{\partial L}{\partial x_{, a b}}\right] n_{a} n_{b}=0 .}
\end{gathered}
$$

Here $n_{a}$ is the unit vector normal to $\Sigma$ and facing the + direction. The first equation (4) is standard representing the balance of linear momentum. The second equation (5) is specific for high gradient theories ensuring the balance of hyper-momentum [47]. 
The Hadamard geometric and kinematic compatibility conditions on $\Sigma$ ensuring the continuity of particle trajectories can be written in the form $\left[x_{, a}\right]=\mu n_{a}$, where $\mu$ is a scalar. Note that the spatial $n_{1}$ and the temporal $n_{2}$ components of the normal vector to $\Sigma$ are related through $n_{2}=-n_{1} V$ where $V$ is the Lagrangian velocity of the discontinuity. Eliminating $\mu$ we obtain the relation $V\left[x_{, 1}\right]+\left[x_{, 2}\right]=0$ representing the balance of mass balance across the discontinuity.

Finally, the Noether identities (3) on $\Sigma$ reduce to

$$
\left[x_{, b} \frac{\delta L}{\delta x_{, a}}+x_{, c b} \frac{\partial L}{\partial x_{, c a}}-L \delta_{b}^{a}\right] n_{a}=0 .
$$

These two equations represent the energy balance and the Bernoulli-Eshelby configurational force balance on the discontinuity. In contrast to (4), (5), none of the relations (6) can be expected to satisfy on the discontinuities bin view of their inherently dissipative nature $[7,52]$.

Unregularized system. To recover the underlying hyperbolic system, we now drop the dependence of $L$ on second derivatives and additively decompose the Lagrangian density into the kinetic and internal energy related parts:

$$
L=\frac{u^{2}}{2}-e(w) .
$$

Here we introduced special notations for the derivatives $w(q, t)=x_{q}$ (strain) and $u(q, t)=x_{t}$ (velocity) implying that the deformation is now written as $x=x(q, t)$ where $q$ is the (mass) Lagrangian coordinate; from now on a subscript without a comma will indicate derivative. The relation between the Eulerian and Lagrangian descriptions is detailed in Appendix A.

In the case when the Lagrangian density is given by (7) the main system, which includes the linear momentum balance equation and the mass balance equation, is known as the $p$-system. It can be written in the form

$$
w_{t}-u_{q}=0, \quad u_{t}+p_{q}=0,
$$

where $p(w)=-e_{w}$. For instance, in $1 \mathrm{D}$ compressible hydrodynamics $w$ is the specific volume, $u$ is the fluid velocity, $e(w)$ is the energy density and $p(w)$ is the fluid pressure $[6,52]$. From now on we assume that $e_{w w}>0$ which makes the system (8) strictly hyperbolic with the characteristic speeds $c_{ \pm}= \pm \sqrt{e_{w w}}$.

Choosing $b=2$ in (3) we obtain the energy conservation law

$$
\left(\frac{u^{2}}{2}+e\right)_{t}+(p u)_{q}=0,
$$

while the component $b=1$ of (3) gives the Bernoulli-Eshelby conservation law

$$
(w u)_{t}+\left(e+w p-\frac{u^{2}}{2}\right)_{q}=0 .
$$

Both equations (9) and (10) are satisfied identically for smooth solutions of the system (8).

Discontinuous solutions of (8) must satisfy the mass conservation and the linear momentum conservation conditions:

$$
\begin{gathered}
V[w]+[u]=0, \\
-V[u]+[p]=0,
\end{gathered}
$$


where again $V$ is the Lagrangian shock velocity. The Noether jump identities (9) and (10) are equivalent and can be written as a single condition

$$
\left[\frac{V^{2} w^{2}}{2}+e+p w\right]=0
$$

Note again that for classical shocks in hyperbolic systems the variational condition (13) is not satisfied.

Regularized system. When both space and time micro-scales are taken into account in a conservative setting we obtain a more general Lagrangian:

$$
\tilde{L}=\frac{u^{2}}{2}-\tilde{e}\left(w, w_{t}, w_{q}\right),
$$

where $\tilde{e}(w, 0,0)=e(w)$. In such model the mass and linear momentum balance equations remain the same as in the associated $p$-system, however the local constitutive relation for the 'pressure' is replaced by

$$
p=-\left(\frac{\partial \tilde{e}}{\partial w}-\frac{\partial}{\partial t}\left(\frac{\partial \tilde{e}}{\partial w_{t}}\right)-\frac{\partial}{\partial q}\left(\frac{\partial \tilde{e}}{\partial w_{q}}\right)\right) .
$$

The energy balance takes the form

$$
\left(\varepsilon+\frac{u^{2}}{2}\right)_{t}+\left(p u-w_{t} \frac{\partial \tilde{e}}{\partial w_{q}}\right)_{q}=0
$$

where we introduced a new energy density $\varepsilon=\tilde{e}-w_{t} \frac{\partial \tilde{e}}{\partial w_{t}}$. The corresponding Bernoulli-Eshelby conservation law reads

$$
\left(w u-w_{q} \frac{\partial \tilde{e}}{\partial w_{t}}\right)_{t}-\left(\frac{u^{2}}{2}-w p+w_{q} \frac{\partial \tilde{e}}{\partial w_{q}}-\tilde{e}\right)_{q}=0
$$

with (16) and (17) remaining equivalent for smooth motions.

Jump discontinuities in the regularized model must respect the generalized RH relations (11) and (12) which remain the same. The two Noether jump conditions again reduce to a single equality which also maintains its form. The only new condition is (5) which we can write in the form

$$
V\left[\frac{\partial \tilde{e}}{\partial w_{t}}\right]=\left[\frac{\partial \tilde{e}}{\partial w_{q}}\right] .
$$

In the case of purely spatial regularization, when $\tilde{e}=\tilde{e}\left(w, w_{q}\right)$, an analog of this condition was obtained in [64], while in the case of purely temporal regularization, when $\tilde{e}=\tilde{e}\left(w, w_{t}\right)$, a special form of (18) was found in [21]. In the latter case, which is of main interest to us here, the condition (18) simplifies to

$$
V\left[\frac{\partial \tilde{e}}{\partial w_{t}}\right]=0 .
$$


Zero dispersion limit. While we now have all the necessary conditions on dispersive discontinuities, their relevance remains to be demonstrated. Below we use these conditions to construct 'composite' TW solutions. We first constrain them to satisfy only classical RH balances. We then replace the discontinuities by smoothed transition layers involving half-solitons and satisfying higher order RH conditions. The ensuing smooth solutions are then shown to serve as close approximations of the outcomes of numerical tests showing the emergence of stationary transition fronts. As we conjecture, the actual discontinuities satisfying all $\mathrm{RH}$ conditions arise only in the zero-dispersion limit.

\section{Composite traveling waves}

Periodic microstructures. An important class of microscopically inhomogeneous and macroscopically homogeneous solutions of the regularized $p$-system (8), (15) is comprised of periodic, cnoidal-type TWs of the type $(w(\theta), u(\theta))$, where $\theta=q-\tilde{D} t$ and $\tilde{D}$ is the (phase) velocity of the wave in Lagrangian coordinates. Using this ansatz we obtain a system of ordinary differential equations with two integrals

$$
\tilde{D} w+u=\tilde{D} \bar{w}+\bar{u}, \quad-\tilde{D} u+p=-\tilde{D} \bar{u}+\bar{p},
$$

where the pressure is $p=-\tilde{e}_{w}+\frac{d}{d \theta}\left(\tilde{e}_{w_{q}}-\tilde{D} \tilde{e}_{w_{t}}\right)$. Note also that here $w_{q}=d w / d \theta$ and $w_{t}=$ $-\tilde{D} d w / d \theta$. The 'overbars,' representing constant values, will be later associated with period averaging. The three constants $\bar{w}, \bar{u}, \bar{p}$, instead of two, were introduced for later convenience.

We can similarly integrate the energy balance equation (16) to obtain

$$
-\tilde{D}\left(\varepsilon^{\star}+\frac{u^{2}}{2}\right)+p u=-\tilde{D}\left(\overline{\varepsilon^{\star}}+\frac{\overline{u^{2}}}{2}\right)+\overline{p u},
$$

where we denoted $\varepsilon^{\star}=\tilde{e}-w_{t} \tilde{e}_{w_{t}}-\frac{d w}{d \theta} \tilde{e}_{w_{q}}=\varepsilon-\frac{d w}{d \theta} \tilde{e}_{w_{q}}$ and introduced additional constants $\overline{\varepsilon^{\star}}, \overline{u^{2}}, \overline{p u}$. Using the conservation laws (20), we can re-write (21) in the equivalent form

$$
\varepsilon^{\star}+\left(\bar{p}+\tilde{D}^{2} \bar{w}\right) w-\frac{\tilde{D}^{2}}{2} w^{2}=\overline{\varepsilon^{\star}}-\frac{\tilde{D}^{2}}{2} \overline{w^{2}}+\left(\bar{p}+\tilde{D}^{2} \bar{w}\right) \bar{w}
$$

featuring yet another integration constant $\overline{w^{2}}$. The following identities linking all these constants can be obtained directly from (20)

$$
\begin{aligned}
& \overline{u^{2}}-(\bar{u})^{2}=\tilde{D}^{2}\left(\overline{w^{2}}-(\bar{w})^{2}\right), \\
& \overline{p u}-\bar{p} \bar{u}=\tilde{D}^{3}\left(\overline{w^{2}}-(\bar{w})^{2}\right) .
\end{aligned}
$$

Specializing energy density. To find explicit relations between the integration constants we need to specify the energy density and in what follows we limit our attention to the case of temporal regularization with quadratic dependence of the energy density $\tilde{e}\left(w, w_{t}\right)$ on $w_{t}$. More specifically, we assume that

$$
\tilde{e}\left(w, w_{t}\right)=e(w)+a(w) w_{t}^{2} .
$$


Note that in this case $\varepsilon^{\star}=\varepsilon$. We also assume that the regularization is perturbative in the sense that there is a small parameter $\epsilon$ in front of the $w_{t}^{2}$ term.

To illustrate this choice, consider a rod with circular cross-section of radius $l$ undergoing small longitudinal deformations. Given that $q$ is the mass coordinate (see Appendix A), the linearized energy density of such rod, accounting for transverse inertia $[49,46]$, can be written in the form

$$
\tilde{e}\left(w, w_{t}\right)=\frac{c^{2}}{2}\left(w^{2}-\nu^{2}\left(\frac{l}{c}\right)^{2} w_{t}^{2}\right),
$$

where $w$ is the longitudinal strain, $c=\sqrt{E / \rho_{0}}$ is the characteristic velocity, $\rho_{0}$ is the material density, $E$ is Young's modulus and $\nu$ is Poisson's ratio. The time scale $l / c$ is responsible for the dispersive effects and its perturbative character becomes apparent in the long wave limit when the characteristic wave length $L \gg l$ is used to non-dimensionalize space. In the normalized variables the regularizing term in the energy appears with the small multiplier $\epsilon=(l / L)^{2} \ll 1$.

Another example can be taken from the theory of bubbly fluids [3]. Suppose that the bubbles are spherical with radius $R(t, q)$ and their number per unit mass $n$ is fixed. Assuming that the fluid component is incompressible with the fixed density $\rho_{f}$, and that the compressible gas is polytropic with exponent $\gamma>1$, we can write the specific energy of the mixture in the form $[18,20]$

$$
\tilde{e}\left(R, R_{t}\right)=\frac{4}{3} \pi R^{3} n\left(\frac{p_{0}}{\gamma-1}\left(\frac{R_{0}}{R}\right)^{3 \gamma}-\frac{3 \rho_{f}}{2} R_{t}^{2}\right),
$$

where $p_{0}$ is the equilibrium pressure and $R_{0}$ is the equilibrium bubble radius. Note that the coefficient in front of the regularizing term $R_{t}^{2}$ contains a time scale $R_{0} / \sqrt{p_{0} / \rho_{f}}$ which is inversely proportional to the Minnaert frequency $\omega^{2}=3 \gamma p_{0} /\left(\rho_{f} R_{0}^{2}\right)$. This time scale characterizes small oscillations of a single bubble in a fluid and is ultimately responsible for dispersive effects. Once again, if the non-dimensionalization of space involves the macroscopic characteristic length $L \gg R_{0}$, we are left with the small parameter $\epsilon=\left(R_{0} / L\right)^{2} \ll 1$ which characterizes the volume fraction of gas bubbles.

Our third example, describing propagation of long surface gravity waves, will be discussed in full detail in Section 4.

Four parametric family of TW solutions. In the case of TWs, the role of the perturbative parameter $\epsilon$ in front of the regularizing term in the energy density reduces to re-scaling of the comoving spatial coordinate $\theta$. Therefore, unless we deal with dimensional quantities, we can assume without loss of generality that $\epsilon=1$.

In the case of quadratic temporal regularization and, independently of the structure of the hyperbolic part of the model, the dimensionless equation (22) reduces to

$$
w_{\theta}^{2}=F(w ; A, B, C),
$$

where

$$
A=\frac{\tilde{D}^{2}}{2}, \quad B=\bar{p}+\tilde{D}^{2} \bar{w}, \quad C=\bar{\varepsilon}-\frac{\tilde{D}^{2}}{2} \overline{w^{2}}+\left(\bar{p}+\tilde{D}^{2} \bar{w}\right) \bar{w} .
$$

The function $F(w)$ depends on the structure of the dispersive model.

Suppose that there is a domain of parameters $A, B, C$ where $F(w)$ has two simple zeros $0<$ $w_{1}<w_{2}$ while remaining positive in the interval between these two roots. Then (25) has periodic 
solutions and the constants $\bar{w}, \bar{w}^{2}, \bar{p}, \bar{\varepsilon}$ can be interpreted as the corresponding period averaged quantities. These parameters can be then expressed as functions of the three constants $A, B, C$, see for instance $[18,19]$. Given that the constant $\bar{u}$ does not enter $(25)$, we conclude that the whole family of TW solutions is determined by four independent parameters.

Dynamic coexistence of wave trains. Consider now the case when a dispersive discontinuity moving in Lagrangian coordinates with a constant velocity $V$ separates two different wave trains. The integration constants, defining each of these wave trains, have to be appropriately matched on the discontinuity surface.

Note first that for the composite wave train to be a TW, the velocity $V$ must coincide with the (phase) velocities of both coexisting TW solutions:

$$
V=\tilde{D}^{-}=\tilde{D}^{+}
$$

In other words both microstructures must be 'frozen' inside the structure of the macroscopic shock.

The mass and linear momentum conservation on the shock can be written in terms of the period averaged quantities:

$$
[V \bar{w}+\bar{u}]=0, \quad[-V \bar{u}+\bar{p}]=0 .
$$

The energy balance equation (16) reads

$$
\left[-V\left(\bar{\varepsilon}+\frac{\bar{u}^{2}}{2}\right)+\bar{p} \bar{u}+\frac{V^{3}}{2}\left(\overline{w^{2}}-(\bar{w})^{2}\right)\right]=0 .
$$

While neither the energy balance, nor the Bernoulli-Eshelby conservation law (17) have to be satisfied on the discontinuity, they remain equivalent for the shocks which satisfy condition (26).

If we now group the two conditions (26) with the two $\mathrm{RH}$ conditions (27), we obtain four equations for the four unknowns defining the state after the shock (given that the state ahead of the shock is known). The still missing constraint on the shock velocity $V$ is delivered by the higher order RH condition (19)

$$
\left[\frac{\partial \tilde{e}}{\partial w_{t}}\right]=0 .
$$

It is appropriate to mention here that while the nonlinear RH conditions associated with the energy balance and the equivalent Bernoulli- Eshelby condition cannot be warranted due to the inevitable dissipation on singularities (due to macro-micro channeling), the linear higher-order RH condition (28), being a direct analog of the momentum balance, should necessarily hold.

Cold-hot discontinuity. Consider now a special case of a dispersive discontinuity between a degenerate state '-', with an infinite wave length ('cold' state), and a non-degenerate periodic wave train representing state '+' with finite wave length ('hot' state). While in the 'hot' state we must require that $V=\tilde{D}^{+}$, where $\tilde{D}^{+}$is the phase velocity of the wave train, in the 'cold' state, the parameter $\tilde{D}^{-}$should be understood as the velocity of the solitary wave $V_{s}^{-}=V$ with the homogeneous configuration '-' as a background. Solitary waves can be viewed as infinite wavelength limits of the wave trains and therefore an appropriate 'half soliton' would naturally interpolate a homogeneous 'cold' state and a periodic 'hot' state. At finite $\epsilon$ the resulting composite construction can be, of course, viewed only as an approximation with the real 'cold-hot' discontinuity emerging in the limit $\epsilon \rightarrow 0$. 
Generalized Riemann problem. The classical Riemann problem for a hyperbolic $p$-system allows only for 'cold' states in the initial conditions [6, 52]. Here we extend this definition for the case of a regularized system. The corresponding generalized Riemann problem can have initial conditions with two bordering macroscopically homogeneous but microscopically inhomogeneous states. This allows, in particular, for the initial coexistence of two 'hot' states. In Section 5 we study such a generalized Riemann problem numerically in the special case when the initial data contain an isolated 'cold-hot' discontinuity. In the whole generality such Riemann problems can be studied in the framework of the averaged Whitham model $[26,27,11,12,14]$.

\section{Serre-Green-Naghdi model}

Due to its many applications, we have chosen the Serre-Green-Naghdi (SGN) model of dispersive gravity waves as an illustrative example for our general theory. The SGN model can be obtained by averaging the free-surface incompressible Euler equations over the depth while keeping only the first order terms in $\epsilon=(d / L)^{2} \ll 0$, where $d$ is the mean depth and $L$ is a characteristic wave length. The rigorous derivation of the SGN model can be found in $[43,36,50]$; the numerical approaches are discussed in $[42,39,17,10]$.

The main system. As it is usual in fluid mechanics, we use Eulerian spatial coordinate $x$ instead of the mass Lagrangian coordinate $q$, see Appendix A for the detailed mapping. The two main unknown functions are the fluid level $h(x, t)$ (replacing the strain $w$ ) and the fluid velocity averaged over the depth $u(x, t)$. Adding to the list of dimensional constants the acceleration of gravity $g$ we can introduce non-dimensional independent variables $\tilde{t}=(\sqrt{g d} / L) t, \tilde{x}=x / L$, and dependent variables $h(t, x)=d \tilde{h}(\tilde{t}, \tilde{x}), u(t, x)=\sqrt{g} d \tilde{u}(\tilde{t}, \tilde{x})$. The SGN system takes the form

$$
\tilde{h}_{\tilde{t}}+(\tilde{h} \tilde{u})_{\tilde{x}}=0, \quad(\tilde{h} \tilde{u})_{\tilde{t}}+\left(\tilde{h} \tilde{u}^{2}+\frac{\tilde{h}^{2}}{2}+\frac{\epsilon}{3} \tilde{h}^{2} \frac{d^{2} \tilde{h}}{d \tilde{t}^{2}}\right)_{\tilde{x}}=0
$$

where $\frac{d^{2} \tilde{h}}{d \tilde{t}^{2}}=\frac{d}{d \tilde{t}}\left(\frac{d \tilde{h}}{d \tilde{t}}\right)$ and $\frac{d \tilde{h}}{d \tilde{t}}=\tilde{h}_{\tilde{t}}+\tilde{u} \tilde{h}_{\tilde{x}}$. Note that the small parameter $\epsilon$ in (29) is proportional to the fourth power of the ratio of the two characteristic time scales $\sqrt{L / g}$ and $L / \sqrt{g d}$. When $\epsilon$ is sufficiently small the dispersive term can be neglected and the SGN system (29) reduces to the classical Saint-Venant system; the latter is a hyperbolic and genuinely nonlinear $p$-system with characteristics propagating with velocities $\tilde{u} \pm \sqrt{\tilde{h}}[52,7]$.

Going back to dimensional variables and assuming that $\epsilon=1$ we obtain the system of equations $[51,59,23,24]$

$$
h_{t}+(h u)_{x}=0, \quad(h u)_{t}+\left(h u^{2}+p\right)_{x}=0
$$

where we introduced the pressure integrated over the fluid depth $p=\frac{g h^{2}}{2}+\frac{1}{3} h^{2} \frac{d^{2} h}{d t^{2}}$. The energy conservation law takes the form

$$
\left(h\left(\frac{u^{2}}{2}+\varepsilon\right)\right)_{t}+\left(h u\left(\frac{u^{2}}{2}+\varepsilon\right)+p u\right)_{x}=0,
$$


where $\varepsilon=\frac{1}{6}\left(\frac{d h}{d t}\right)^{2}+\frac{g h}{2}$. The associated Lagrangian is $[45,20]$

$$
L=h\left(\frac{u^{2}}{2}+\frac{1}{6}\left(\frac{d h}{d t}\right)^{2}-\frac{g h}{2}\right) .
$$

In terms of the variable $w(q, t)=h^{-1}$, used in (14), the internal energy density is

$$
\tilde{e}\left(w, w_{t}\right)=\frac{g}{2 w}-\frac{w_{t}^{2}}{6 w^{4}} .
$$

Note that in this case the regularizing term depends not only on $w_{t}$ but also on $w$.

Periodic TWs. A detailed description of the TW solutions to SGN equations (30) can be found in [12]. We summarize it here in the form suitable for further analysis.

Suppose that $h=h(\xi), u=u(\xi)$, where $\xi=x-D t$ and $D$ is a constant wave velocity. From (30) we obtain

$$
\begin{gathered}
h(u-D)=m, \\
p+\frac{m^{2}}{h}=\iota,
\end{gathered}
$$

where we introduced the constants $m$ and $\iota$. Equation (33) can be rewritten as

$$
\frac{1}{2} g h^{2}+\frac{1}{3} m^{2} h\left(\frac{h^{\prime}}{h}\right)^{\prime}+\frac{m^{2}}{h}=\iota .
$$

It has a first integral

$$
\frac{1}{6}\left(\frac{h^{\prime}}{h}\right)^{2}+\frac{g h}{2 m^{2}}-\frac{1}{2 h^{2}}+\frac{\iota}{m^{2} h}=e
$$

where $e$ is another integration constant. The equation (34) can be recast in the form

$$
\left(h^{\prime}\right)^{2}=F(h),
$$

with $F(h)=-\frac{3 g}{m^{2}} h^{3}+6 e h^{2}-\frac{6 \iota}{m^{2}} h+3=\frac{3 g}{m^{2}} P(h)$. In terms of the roots of the polynomial $P(h)=\left(h-h_{0}\right)\left(h-h_{1}\right)\left(h_{2}-h\right)$, where $0<h_{0} \leq h_{1}<h_{2}<\infty$, the integration constants $m^{2}, \iota$ and $e$ can be expressed explicitly

$$
m^{2}=g h_{0} h_{1} h_{2}, \quad \iota=\frac{g}{2}\left(h_{0} h_{1}+h_{0} h_{2}+h_{1} h_{2}\right), \quad e=\frac{h_{0}+h_{1}+h_{2}}{2 h_{0} h_{1} h_{2}} .
$$

The periodic (cnoidal) solutions exist in the domain $h_{0}<h_{1}<h_{2}$ with oscillations between the values $h_{1}$ and $h_{2}$. It can be written explicitly in the form:

$$
h(\xi)=h_{1}+\left(h_{2}-h_{1}\right) \mathrm{cn}^{2}(\kappa \xi ; s), \quad u(\xi)=D+\frac{m}{h(\xi)},
$$

where $\kappa^{2}=\frac{3}{4} \frac{\left(h_{2}-h_{0}\right)}{h_{0} h_{1} h_{2}}, s^{2}=\frac{h_{2}-h_{1}}{h_{2}-h_{0}}$. Note that the sign of the mass flux $m= \pm \sqrt{g h_{0} h_{1} h_{2}}$ is chosen to be negative (positive) for right (left)-facing waves. The Jacobi elliptic function $c n(u ; s)$ is 
defined by the equality $\operatorname{cn}(v ; s)=\cos (\varphi(v, s))$, where the function $\varphi(v, s)$ can be obtained implicitly from the relation $\int_{0}^{\varphi}\left(1-s^{2} \sin ^{2}(\theta)\right)^{-1 / 2} d \theta=v$. The wave length can be found from the constraint

$$
\lambda=2 \int_{h_{1}}^{h_{2}} \frac{d h}{\sqrt{F\left(h ; h_{0}, h_{1}, h_{2}\right)}} .
$$

The relation between the special set of parameters $h_{0}, h_{1}$ and $h_{2}$, used here, and the set $A, B$, $C$, used in the general theory, can expressed explicitly in terms of complete elliptic integrals [12]. As we have already mentioned, the whole family of such solutions is four-parametric and is fully defined, for instance, by the constants $h_{0}, h_{1}, h_{2}$ and $D$.

Solitary waves. In the infinite wavelength limit the above construction produces isolated solitary waves. The solution of (35) corresponding to $\lambda=\infty$ is selected by the condition $h_{0}=h_{1}$ and can be written explicitly

$$
h(\xi)=h_{1}+\left(h_{2}-h_{1}\right) \operatorname{sech}^{2}\left(\frac{\xi}{2} \sqrt{\frac{3\left(h_{2}-h_{1}\right)}{h_{2} h_{1}^{2}}}\right), \quad u(\xi)=D+\frac{m}{h(\xi)}, \quad m=h_{1}\left(u_{1}-D\right) .
$$

Here $h_{1}, h_{2}$ are the fluid levels at infinity and under the soliton's crest, while $u_{1}$ is the velocity at infinity. Under the assumption that the solitary wave moves to the right we obtain $m=-h_{1} \sqrt{g h_{2}}$ and therefore

$$
D=u_{1}+\sqrt{g h_{2}} \text {. }
$$

The whole family of such solutions is three-parameteric and is fully defined by the constants $h_{1}, h_{2}$ and $D$.

Admissible 'hot-to-cold' transitions. From now on we use the notation $\star$ to indicate the 'cold' state. Assuming first that it is homogeneous with $h \equiv h_{\star}, u \equiv u_{\star}$ we can write the RankineHugoniot relations for the mass and linear momentum in the form

$$
\begin{gathered}
h u=h_{\star} u_{\star}=m, \\
\frac{m^{2}}{h}+\frac{g h^{2}}{2}+\frac{m^{2}}{3} \frac{d^{2} h}{d x^{2}}-\frac{m^{2}}{3 h}\left(\frac{d h}{d x}\right)^{2}=\frac{m^{2}}{h_{\star}}+\frac{g h_{\star}^{2}}{2}=\iota .
\end{gathered}
$$

Here we have already used the assumption $V^{E}=D=0$, where $V^{E}$ stands for the Eulerian shock velocity. Under this assumption, the additional condition (19) reduces in the Eulerian coordinates to

$$
m^{2}\left[\frac{d h}{d x}\right]=0
$$

Since in the homogeneous state $\frac{d h}{d x} \equiv 0$ the condition (43) suggests that a 'hot-to-cold' discontinuity must necessarily involve the extremas $h_{1}, h_{2}$ of the 'hot' state. 


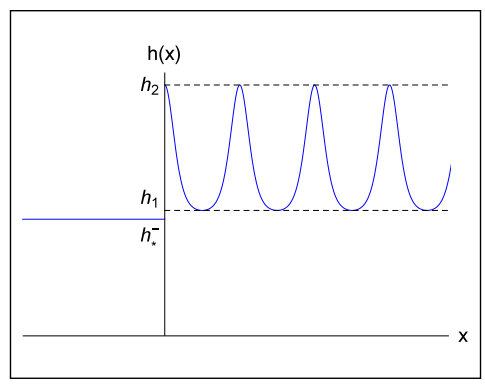

Figure 1: Schematic representation of a discontinuity connecting a homogeneous state $h \equiv h_{\star}$ to a periodic wave train at its maximum point $h=h_{2}$.

We now show that there are only two homogeneous states $h_{\star}^{ \pm}$which can be connected to a stationary wave train through its extremal points $h_{1}$ and $h_{2}$. Indeed, from the RH conditions (41) - (42) and the relations (36) we obtain that $h_{\star}$ must be a root of the third order polynomial:

$$
Q\left(h_{\star}\right)=h_{\star}^{3}-\left(h_{0} h_{1}+h_{0} h_{2}+h_{1} h_{2}\right) h_{\star}+2 h_{0} h_{1} h_{2} .
$$

Since $h_{0}<h_{1}<h_{2}$, the polynomial (44) always has three real roots $h_{\star}$ : one is negative, and two others $h_{\star}^{-}<h_{\star}^{+}$are positive, moreover,

$$
h_{0}<h_{\star}^{-}<h_{1}<h_{\star}^{+}<h_{2},
$$

see Appendix B for details. The shock connecting the states $h_{\star}^{-}$and $h_{2}$ is schematically shown in Fig. 1.

It can be also shown by direct computation that for the right facing stationary wave with $m<0$ and $D=0$ the inequalities

$$
u_{\star}^{-}+\sqrt{g h_{\star}^{-}}<0, u_{\star}^{-}-\sqrt{g h_{\star}^{-}}<0,
$$

and

$$
u_{\star}^{+}+\sqrt{g h_{\star}^{+}}>0, u_{\star}^{+}-\sqrt{g h_{\star}^{+}}<0,
$$

are necessarily satisfied, see Appendix B. These inequalities, specifying the slopes of the shocks in relation to the slopes of the incoming and outcoming of characteristics of the non-regularized system, will be interpreted later.
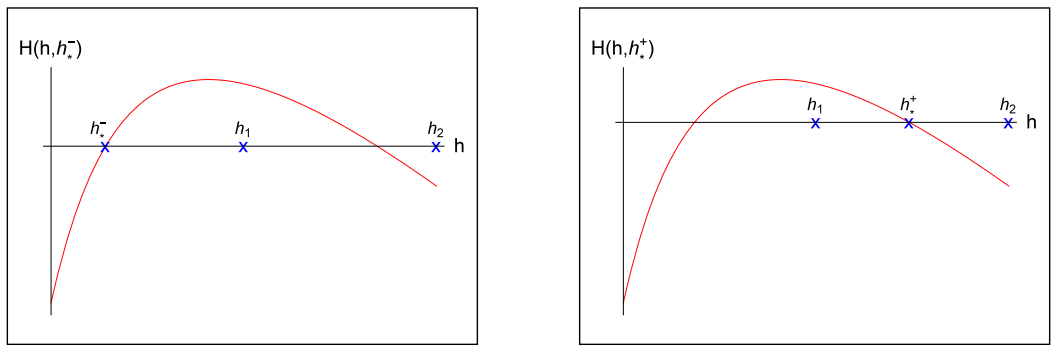

Figure 2: Qualitative behavior of the functions $H\left(h, h_{\star}^{ \pm}\right)$. 
To distinguish between the four eligible connections $h_{1,2} \rightarrow h_{\star}^{ \pm}$consider the function

$$
H\left(h, h_{\star}\right)=\frac{h_{0} h_{1} h_{2}}{2 h_{\star}^{2}}+h_{\star}-\frac{h_{0} h_{1} h_{2}}{2 h^{2}}-h .
$$

The equation $H\left(h_{i}, h_{\star}\right)=0$ is equivalent to the condition of energy conservation on the discontinuity

$$
\frac{u^{2}}{2}+g h+\frac{m^{2}}{3 h} \frac{d^{2} h}{d x^{2}}-\frac{m^{2}}{6 h^{2}}\left(\frac{d h}{d x}\right)^{2}=\frac{m^{2}}{2 h_{\star}^{2}}+g h_{\star} .
$$

The qualitative behavior of the function $H\left(h, h_{\star}^{ \pm}\right)$is illustrated in Fig. 2. One can see that the energy is lost at the discontinuities $h_{2} \rightarrow h_{\star}^{ \pm}$and is acquired at the discontinuities $h_{1} \rightarrow h_{\star}^{ \pm}$which makes the latter energetically inadmissible.

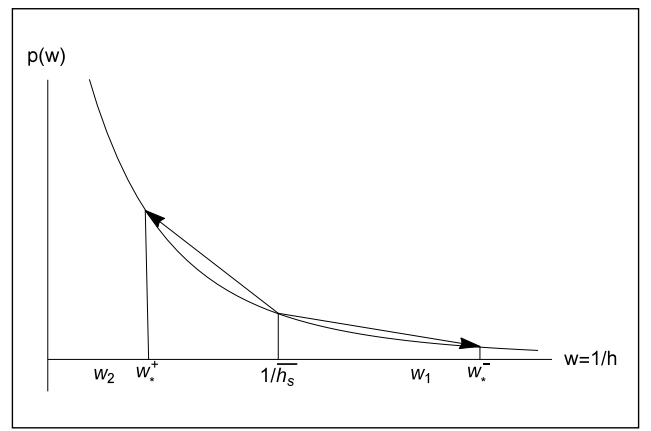

Figure 3: Schematic diagram showing the non-admissible expansion shock connecting the averaged state $h=\overline{h_{s}}$ with the homogeneous state $h=h_{\star}^{-}$and the admissible compression shock connecting the homogeneous state $h=\overline{h_{s}}$ with the homogeneous state $h=h_{\star}^{+}$.

To separate the two remaining options $h_{2} \rightarrow h_{\star}^{ \pm}$it is instructive to look at these shocks from the perspective of the hyperbolic theory (St-Venant equations). The latter deals with period averaged configurations. While for the 'cold' state it is the actual configuration, for the 'hot' state we need to define the virtual homogeneous state

$$
h \equiv \bar{h}_{s}\left(h_{0}, h_{1}, h_{2}\right)=h_{0}+\left(h_{2}-h_{0}\right) \frac{E(s)}{K(s)},
$$

where $K(s)=\int_{0}^{\frac{\pi}{2}}\left(1-s^{2} \sin ^{2} \theta\right)^{-1 / 2} d \theta$ and $E(s)=\int_{0}^{\frac{\pi}{2}}\left(1-s^{2} \sin ^{2} \theta\right)^{1 / 2} d \theta$ are the complete elliptic integrals of the first and second type with the modulus $s^{2}=\frac{h_{2}-h_{1}}{h_{2}-h_{0}}$. In terms of such averaged quantities and the corresponding hyperbolic theory, the transition $\bar{h}_{s} \rightarrow h_{\star}^{-}$is an inadmissible (expansion shock) while the transition $\bar{h}_{s} \rightarrow h_{\star}^{+}$is admissible (compression shock), see Fig. 3 .

Indeed, consider the structure of characteristics around each of these shocks. In the case of the transition $\bar{h}_{s} \rightarrow h_{\star}^{-}$we have $u_{\star}^{-}+\sqrt{g h_{\star}^{-}}<0$ and $u_{\star}^{-}-\sqrt{g h_{\star}^{-}}<0$. Therefore both characteristics in the state with $h \equiv h_{\star}^{-}$have negative slopes, see Fig. 4 (left figure). The characteristics in the state with $h \equiv \bar{h}_{s}$ are presented in this figure under the special assumption that $\bar{u}_{s}=-\sqrt{g \bar{h}_{s}}$ 
which will be justified below. With only two characteristics 'coming' to the front it becomes 'undercompressive' and therefore unstable [63]. For the transition $\bar{h}_{s} \rightarrow h_{\star}^{+}$the pattern of characteristics is different, see Fig. 4 (right figure). In this case the characteristics in the state $h \equiv h_{\star}^{-}$have velocities of opposite signs : $u_{\star}^{+}+\sqrt{g h_{\star}^{+}}>0$ and $u_{\star}^{+}-\sqrt{g h_{\star}^{+}}<0$, while the configuration of the characteristics in the state $h \equiv \bar{h}_{s}$ remains the same. We see that now three characteristics are coming and one is leaving, which is the classical configuration for a stable shock wave [63].

In the regularized problem the above stability assessment must be reversed because of the presence of an additional RH condition. In particular, the under-compressive shocks $\bar{h}_{s} \rightarrow h_{\star}^{-}$ become stabilized while the stability of the classical shocks $\bar{h}_{s} \rightarrow h_{\star}^{+}$can no longer be guaranteed. Our numerical experiments, reported in Section 5, support this theoretical prediction.
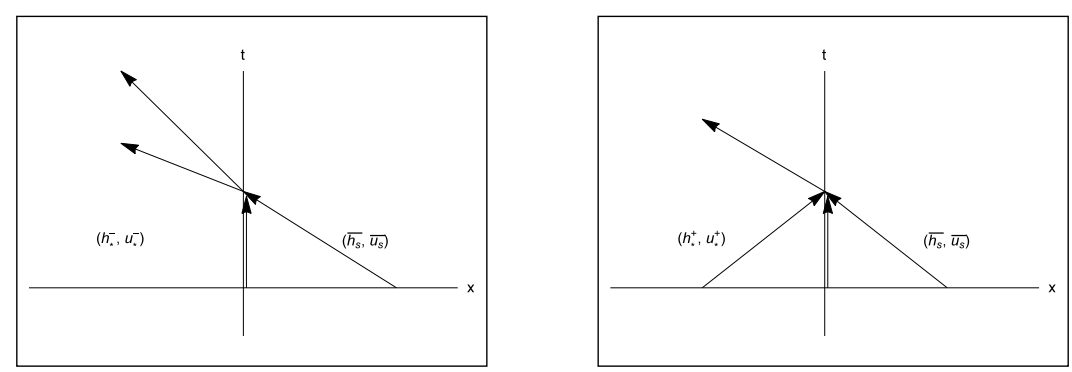

Figure 4: Schematic structure of characteristics in the hyperbolic problem for the stationary expansion shock connecting the homogeneous state $h \equiv \bar{h}_{s}$ on the right with the homogeneous state $h \equiv h_{\star}^{-}$on the left (left figure), and for the stationary compressive shock connecting the homogeneous state $h \equiv \bar{h}_{s}$ on the right with the homogeneous state $h \equiv h_{\star}^{+}$on the left (right figure).

Interpolation. At finite $\epsilon$ the admissible discontinuous transitions $\bar{h}_{s} \rightarrow h_{\star}^{-}$do not formally belong to the family of composite periodic TWs because the constant state $h \equiv h_{\star}^{-}$is different from the periodic TW with $\lambda=\infty$ which must be a solitary wave. The solitary wave, compatible with the transition $\bar{h}_{s} \rightarrow h_{\star}^{-}$, must have the background $h_{\star}^{-}$and the maximum $h=h_{2}$. A half-soliton with these parameters will smoothly interpolate between the homogeneous state $h \equiv h_{\star}^{-}$and an infinite wave train with the average $\bar{h}_{s}$, see Fig. 5 (left figure). The background velocity in such solitary wave is $u_{1}=-\sqrt{g h_{2}}$.
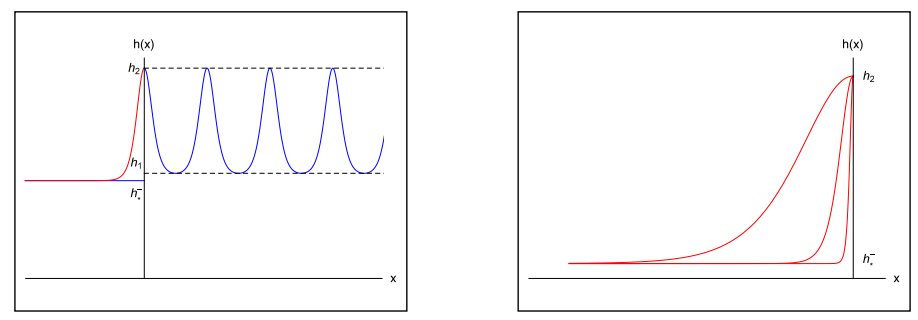

Figure 5: Left figure: half-soliton interpolating between a constant state $h_{\star}^{-}$and a periodic wave train with the maximum at $h_{2}$ at $\epsilon=1$ (shown in red). Right figure: sharpening of such interpolation as the scaling parameter $\epsilon$ tends to zero. 
The obtained composite configuration, however, is not a TW solution of the system (30), because the equation, describing the TW solutions, is of the second order, and it does not have heteroclinic orbits connecting periodic and homogeneous states. Thus, our composite wave train can be considered only as an approximation to the actual solution which requires for its construction a more complex anzatz containing non-TW elements.

Even though at finite values of $\epsilon$ the described composite TW is only an approximation of the actual solution, its $\epsilon \rightarrow 0$ limit (Fig. 5 (right figure)) can be expected to approach the exact discontinuous solution, see Fig. 1. To the best of our knowledge, the emergence in the zero dispersion limit of such generalized shocks, linking homogeneous configurations with measure-valued infinitely fine dynamic mixtures, has not been reported before. To understand stability of such shocks it is necessary to study the associated higher order hyperbolic (Whitham) system [60].

Generalized Riemann problem. To illustrate these ideas consider the simplest initial value problem when a non-trivial periodic TW solution is placed in contact with a constant state characterized by the same averaged parameters. Suppose that the real axis is partitioned into the following three domains:

$$
\left(\begin{array}{c}
h(0, x) \\
u(0, x)
\end{array}\right)= \begin{cases}\left(\begin{array}{c}
h_{R}=\overline{h_{s}} \\
u_{R}=0
\end{array}\right), & \text { if } x>L, \\
\left(\begin{array}{c}
h_{s}(x) \\
u_{s}(x)
\end{array}\right), & \text { if }-L<x<L, \\
\left(\begin{array}{c}
h_{L}=\overline{h_{s}} \\
u_{L}=0
\end{array}\right), & \text { if } x<-L .\end{cases}
$$

Here $\left(h_{s}(x), u_{s}(x)\right)$ is a periodic solution of the SGN system, with the average values $\bar{h}_{s}(x)$ and $\bar{u}_{s}(x)=0$. In the non-regularized (Saint-Venant) setting such initial value problem would have had only a trivial solution.

To specify parameters, we need to choose the values $h_{0}, h_{1}, h_{2}$ and select the wave velocity $D$. We first recall that $h_{s}\left(u_{s}-D\right)=m$, and $m=-\sqrt{g h_{0} h_{1} h_{2}}$ where the choice of the sign reflects the fact that the TW moves in Lagrangian coordinates to the right $(D>0)$. We can then write $D=\overline{u_{s}}-m \overline{h_{s}^{-1}}$. Since we can always choose $\overline{u_{s}}=0$ we write $D=-m\left(\overline{h_{s}^{-1}}\right)$. Also, using the Galilean invariance we can choose the coordinate system moving with velocity $D$, which will make the shock stationary in Eulerian coordinates. This will lead to the appearance of a nonzero flow at infinity with $u_{R, L}=-D$.

Under the transformation $x \rightarrow x+D t, u \rightarrow u+D$ the initial data (50) transform into

$$
\left(\begin{array}{c}
h(0, x) \\
u(0, x)
\end{array}\right)=\left\{\begin{array}{l}
\left(\begin{array}{c}
h_{R}=\overline{h_{s}} \\
u_{R}=m\left(\overline{h_{s}^{-1}}\right)
\end{array}\right), \quad \text { if } x>L, \\
\left(\begin{array}{c}
h_{s}(x) \\
u_{s}(x)+m\left(\overline{h_{s}^{-1}}\right)
\end{array}\right), \quad \text { if }-L<x<L, \\
\left(\begin{array}{c}
h_{L}=\overline{h_{s}} \\
u_{L}=m\left(\overline{h_{s}^{-1}}\right)
\end{array}\right), \quad \text { if } x<-L .
\end{array}\right.
$$


Choosing $m$ and $\overline{h_{s}}$ we fix two of the three parameters $h_{0}, h_{1}, h_{2}$. The choice of the third parameter remains unconstrained.

Sonic Riemann data. Our numerical experiments show that a generic 'cold-hot' stationary shock splits into a special stationary 'cold-hot' shock and a spreading dispersive shock wave. Since in the configuration of interest the trailing edge of such dispersive shock would move with the sonic velocity of the homogeneous state $\overline{h_{s}}$, the influence of the boundary of the computational domain at $x=-L$ can be eliminated if the initial shock also moves with the same sonic velocity (in Lagrangian coordinates). It is then natural to select the initial conditions respecting the condition $D=\bar{c}_{s}$, where $\overline{c_{s}}=\sqrt{g \bar{h}_{s}}$ is the sonic velocity in the state $h \equiv \overline{h_{s}}$. The ratio $D / \bar{c}_{s}$ is known as the Mach number $M$ (Froude number in hydraulics). In the SGN context we can write (given that $m<0$ )

$$
M=-\frac{m \overline{h_{s}^{-1}}}{\sqrt{g \overline{h_{s}}}} .
$$

Accordingly, our numerical experiments discussed in the next Section were conducted under the assumption that $F r=1$. The ensuing constraint on the parameters $h_{0}, h_{1}, h_{2}$ is illustrated in Fig. 6.

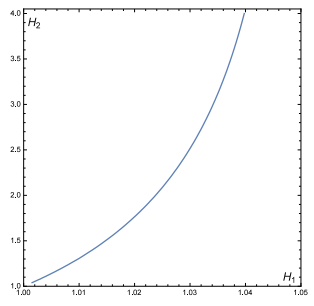

Figure 6: The critical line $M=1$ in the plane $\left(H_{1}, H_{2}\right)$ with $H_{i}=h_{i} / h_{0}$.

\section{$5 \quad$ Numerical solution of a generalized Riemann problem}

To solve such a generalized Riemann problem, we used the numerical method developed for the SGN system in [39]. Its adaptation to our problem is described in detail in Appendix C; the version we actually used is illustrated there for the benchmark test known as the 'dam break problem'.

Test 1. In Figure 7, we show the initial fluid level configuration setting up the generalized Riemann problem (51). The parameters are chosen to satisfy approximately the relation $F r=1$. For computational convenience we connected the initial homogeneous state to the adjacent periodic solution smoothly; the smoothing procedure is discussed in Appendix D.

A snapshot of the numerical solution at $t=1000 \mathrm{~s}$ is shown in Figure 8. Observe the formation of a new homogeneous state (the $\star$ state) between the dispersive shock wave moving to the right in Lagrangian coordinates and the stationary discontinuity which also moves to the right in Lagrangian coordinates effectively consuming the periodic wave train. The distribution of wave lengths (shown in the lower portion of the figure) suggests that the initial wave train remains unperturbed by the breakdown of the original shock. 


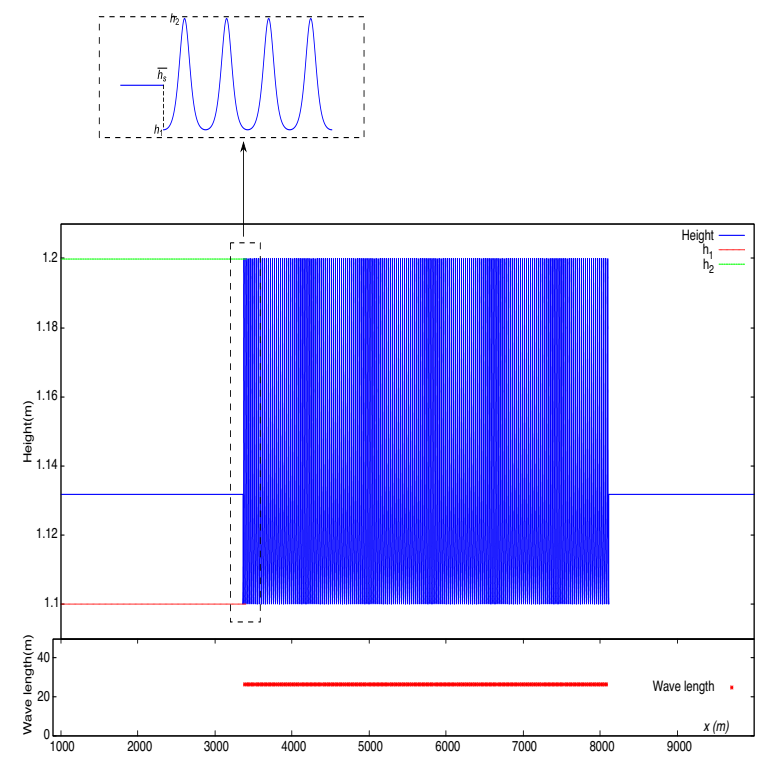

Figure 7: The initial fluid level distribution $h(x, 0)$ described by $(51)$. The periodic wave train parameters are : $h_{0} \approx 1.0962 \mathrm{~m}, h_{1}=1.1 \mathrm{~m}$, and $h_{2}=1.2 \mathrm{~m}, \lambda \approx 26.3767 \mathrm{~m}$. The corresponding period average value of $h$ is $\overline{h_{s}} \approx 1.13173 \mathrm{~m}$. The 'cnoidal-type' wave train in the middle can be closely approximated by an assembly of 180 single solitary waves (37). Inset: a zoom into the transition region on the left. In the actual numerical experiments the initial discontinuity between $\bar{h}_{s}$ and $h_{1}$ was smoothed using the hyperbolic tangent interpolation (63).

The analysis of subsequent snapshots shows that the front separating the emerging $\star$ state and the initial periodic wave train is stationary in Eulerian coordinates. Our insets show that the diffused interpolating layer starts at $h_{\star}^{-}$and ends exactly at the maximum point $h=h_{2}$ of the periodic solution, see Figure 8. The value of velocity $u_{\star}^{-}$is in agreement with the value calculated from the mass conservation law : $u_{\star}^{-} h_{\star}^{-}=-\sqrt{g h_{0} h_{1} h_{2}}$. For the parameters $h_{0} \approx 1.0962 m, h_{1}=1.1$ $m$, and $h_{2}=1.2 m$ chosen in Figure 7 , we obtained the values $h_{\star}^{-} \approx 1.09808$ and $u_{\star}^{-} \approx-3.46416$.

As we have already explained, the spreading of the transition layer between the fluid levels $h=h_{\star}^{-}$and $h_{2}$ is due to the presence of a finite scale $\epsilon=1$ in our problem. With this scale fixed the numerical convergence of the numerical method as the mesh size goes to zero is illustrated in the upper right inset in Figure 8; note in particular the convergence of the limiting values $h_{\star}^{-}$ and $h=h_{2}$ to the analytically predicted values. It can be also seen that the interpolating layer is numerically close to the half of a stationary solitary wave (39), characterized by the conditions $D=0$ and $h_{1}=h_{\star}^{-}$; the velocity of such soliton at infinity is necessarily equal to $u_{\star}^{-}$. 


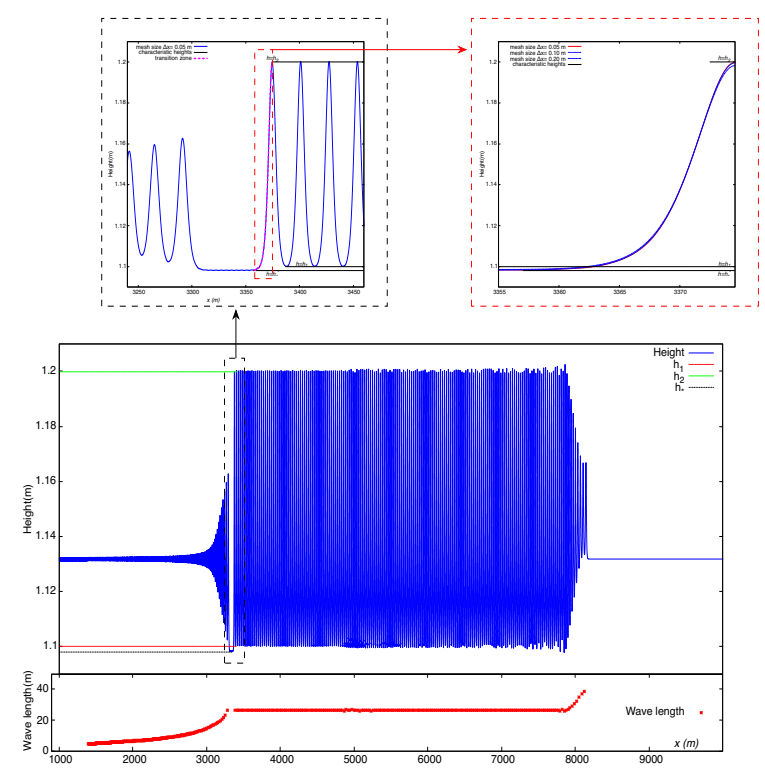

Figure 8: Numerical solution of the generalized Riemann problem (51) at $t=1000 \mathrm{~s}$; the initial conditions are shown in Fig. 7. The spatial distribution of wavelengths (distances between the closest local maxima) is shown in the bottom. The insets show two progressive zooms around the emerging stationary shock.

Test 2. Next, consider the initial data where the homogeneous state on the left is chosen to coincide exactly with the $\star$ configuration so that the value $h=h_{\star}$ appears in (44) as the minimal positive root, see Fig. 9 (the left upper figure). More precisely we choose $h=h_{\star}^{-} \approx 1.09808 \mathrm{~m}$ and $u=u_{\star}^{-} \approx-3.46416 \mathrm{~m} / \mathrm{s}$ while the parameters of the periodic wave train remain as before : $h_{0} \approx 1.0962 \mathrm{~m}, h_{1}=1.1 \mathrm{~m}, h_{2}=1.2 \mathrm{~m}$ and $\mathrm{g}=10 \mathrm{~m} / \mathrm{s}^{2}$. The goal of this numerical experiment is to test the stability of the stationary shock emerging in Test $\mathbf{1}$ and now incorporated directly into the initial data. Once again, we connected the initial state $\star$ to the adjacent periodic solution smoothly as discussed in Appendix D.

The evolution of the solution is illustrated in Fig. 9 at times $t=1000 \mathrm{~s}$ (the right upper figure), $t=2000 \mathrm{~s}$ (the bottom left figure) and $t=6000 \mathrm{~s}$ (the bottom right figure). A quantitative comparison of these snapshots shows that neither the width nor the amplitude of the stationary shock on the left changes with time at the scale of our numerical experiment.

The snapshots at times $t=2000 \mathrm{~s}$, and $t=6000 \mathrm{~s}$ show the sign of small non-stationary fluctuations propagating along the constant state ' $\star$ '. These small-amplitude waves moving to the left are generated on the right extremity of the periodic wave train due a non-stationary process over there (see more about this below). They have already passed the stationary shock-like transition front and will be eventually absorbed by the computational boundary on the left. Despite these external perturbations, the stationary pattern shows remarkable resilience by maintaining its structure and showing only minimal modulation of the states on both sides of the front.

To make sure that the modulation is indeed due exclusively to the waves arriving from the right, and is not revealing the non-stationary nature of the shock-like transition front itself, we performed a series of special numerical experiments. In particular, in an attempt to completely suppress waves 
coming from the right, we initially imposed the state $h=h_{\star}^{-}$both on the left and on the right, see the left upper figure in Figure 10.

In the right upper figure in Figure 10 we show the corresponding solution at time $2000 \mathrm{~s}$. While the global structure of the emerging wave pattern is rather different from the case when the initial constant state on the right was $\bar{h}$, the structure of the stationary shock-like front (solitary wave merging with the 'cnoidal' type wave train) remains exactly the same and we only see a small horizontal shift. The detailed comparison at time $2000 s$ is illustrated in the two bottom figures in Figure 10. In particular, one can see that the superimposed fluctuations of the constant state, consciously present in the test with the state $\bar{h}$ imposed on the right, completely disappear in the test when the state on the right is $h_{\star}^{-}$. All this suggests that the obtained shock-like transition has at least a finite range of stability.
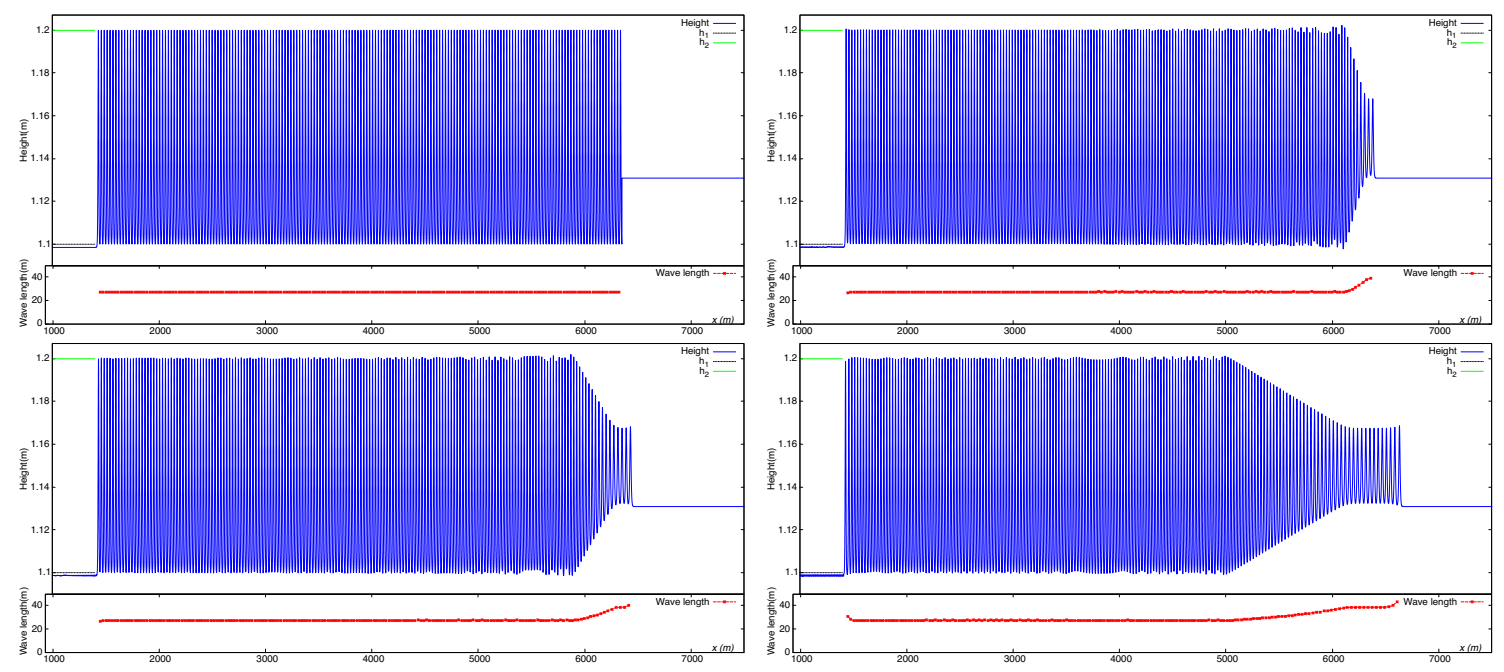

Figure 9: Upper left figure: the initial condition for the generalized Riemann problem with state $h=h_{\star}^{-}$on the left and the same wave train as in Fig. 7 on the right. The numerically obtained snapshots of the solution are shown at three time instants : $t=1000 \mathrm{~s}$ (upper right figure), $t=2000 \mathrm{~s}$ (bottom left figure), and $t=6000 \mathrm{~s}$ (bottom right figure). 

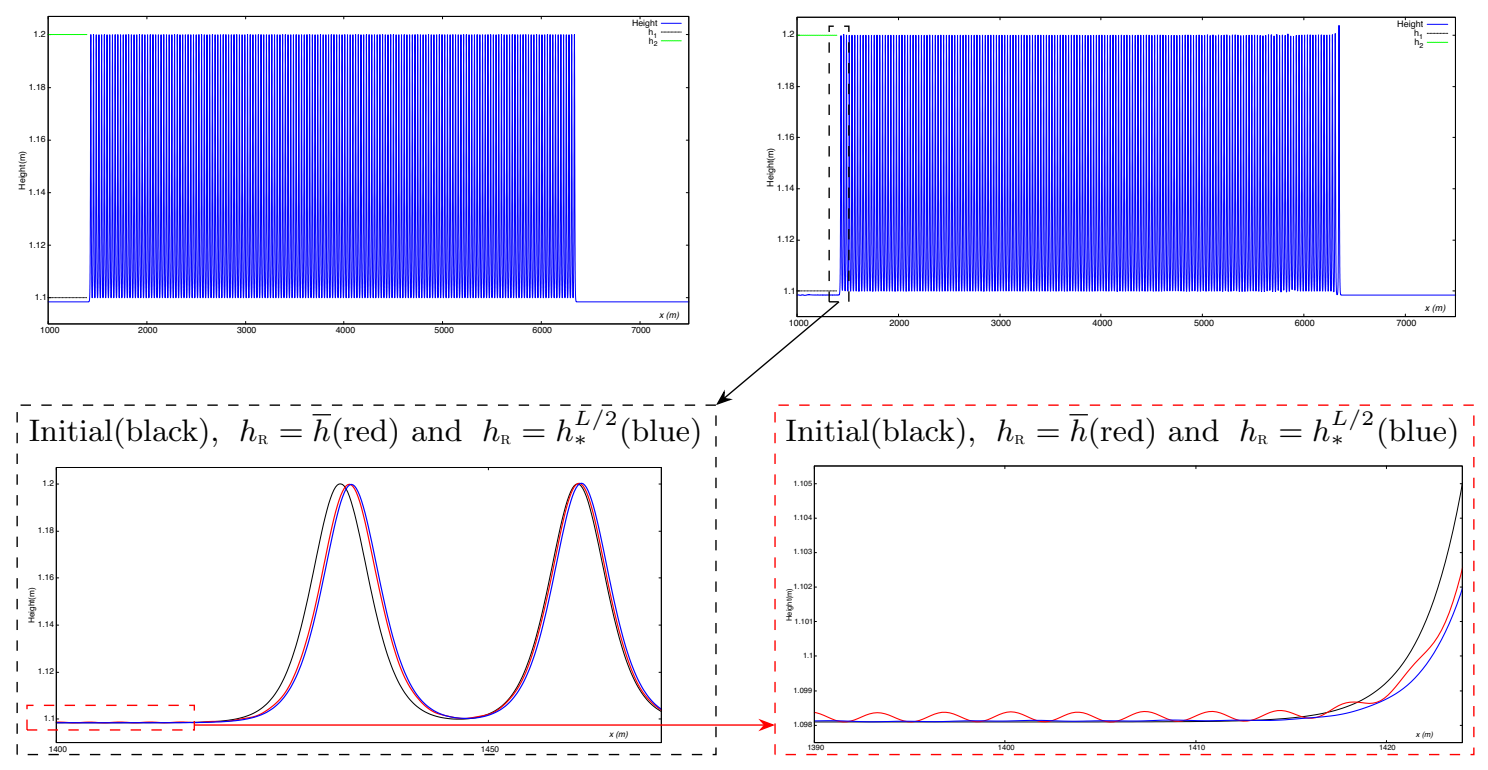

Figure 10: Upper figures : initial conditions showing the state $h_{\star}^{-}$imposed both on the left and on the right (left); the ensuing solution at time $2000 s$ (right). Bottom figures: comparison of the transition front structures generated by the initial conditions with either $h=\overline{h_{s}}$ (red line) or $h=h_{\star}^{-}$(blue line) imposed on the right side of the computational domain.

In Fig. 10 we used the notation $h_{\star}^{L / 2}$ to indicate that in the initial conditions the state $h_{\star}^{-}$ was matched to the periodic wave train using an interpolation over the length $L_{\star} / 2$ (see Appendix D for details of this interpolation). In the test reported in Fig. 10 we used as $L_{\star}$ the length of the periodic wave with parameters $h_{0}, h_{\star}$ and $h_{2}$ which allowed us to relate smoothly the periodic wave train and the state 'star' ( with both the solution and its first derivative continuous). Smaller smoothing scales have been tried as well but they have all lead finally to the creation of the same transition layer with the scale of a half of the solitary wave. To show that the formation of shocklike stationary front does not depend on the interpolation length at all, we present in Fig. 11 the results of the simulations without any interpolation at all, when the initial data were discontinuous. Note the transient adjustment of the solution through the formation of large-amplitude solitonic perturbation with oscillatory tail ahead (moving to the left). However, it clearly leaves behind (on the right) exactly the stationary structure which we discussed above (a one half of a solitary waves connecting smoothly the wave train with the $\star$ state). This is yet another evidence of the numerical stability for the stationary fronts constructed in this paper.

Finally observe that when the boundary condition on the right is $h=\overline{h_{s}}$, the initial periodic wave train ('hot' configuration) progressively transforms on the right extremity into another 'hot' configuration with a larger wave length, see Fig. 9. We can interpret this transition as an expanding dispersive wave which effectively bridges 'more hot' and 'less hot' states. Note that the average value of the variable $h$ in the newly formed wave train is larger than in the original state $h=\overline{h_{s}}$. The ensuing complex breakdown pattern can be potentially explained using the Whitham's averaged equations, for instance, in the zero dispersion limit the observed 'hot-hot' transition is suggestive of a higher dimensional hyperbolic rarefaction wave. 


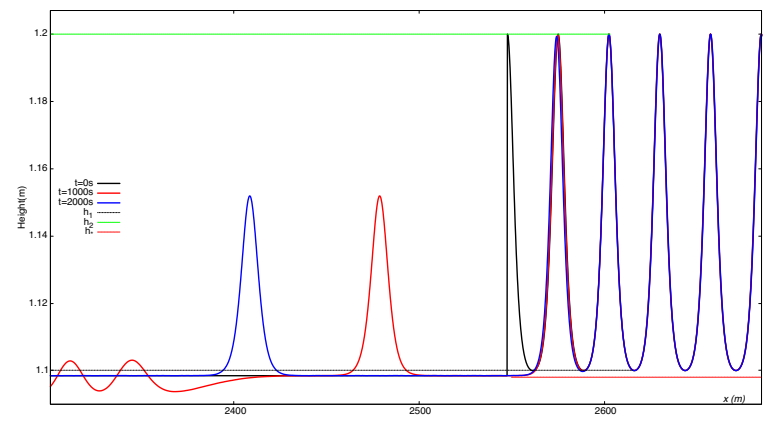

Figure 11: The same initial configuration as in Fig. 10 but without the initial smoothing of the transition between the periodic wave train and the state $\star$. The initial data are shown by the solid black line.

Test 3. Consider next a similar initial configuration where instead of the state $h_{\star}^{-}$we choose the state $h_{\star}^{+}$on the left. In terms of the underlying hyperbolic system we now have initially a compression shock connecting the homogeneous state $h=\bar{h}_{s}$ with the homogeneous state $h=h_{\star}^{+}$, see Fig. 4 (right figure).

As we have already seen, the corresponding pattern of characteristics in the non-regularized hyperbolic problem suggests instability, see Fig. 4 (right figure). In agreement with this prediction, in our numerical experiment the stationary transition front is not forming, see Fig. 12. We observe instead a complex breakdown pattern which requires for its understanding a study of the corresponding Whitham's higher order system.

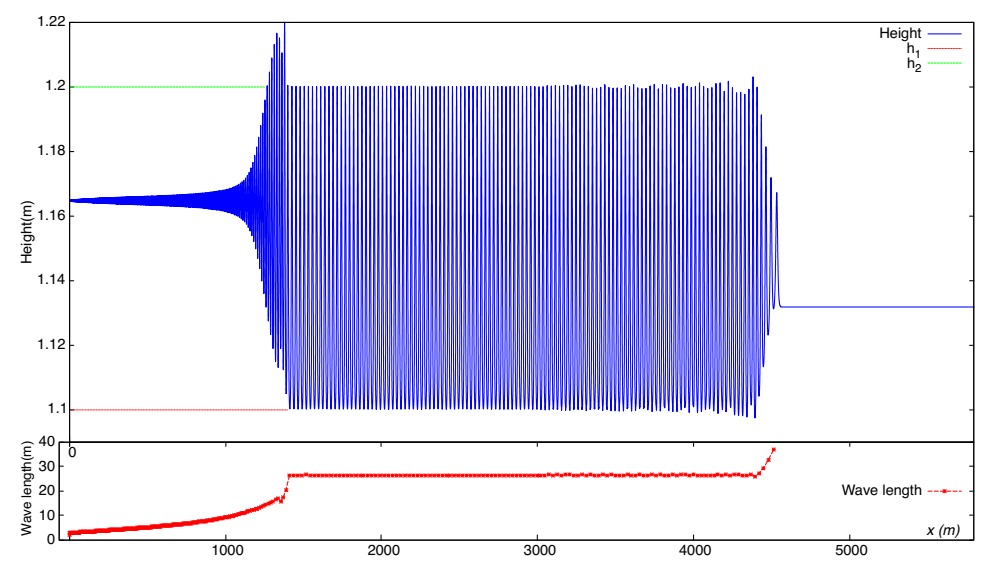

Figure 12: The decomposition of the initial state with $h=h_{\star}^{+} \approx 1.16471$ on the left and the same wave train as in Fig. 7 on the right. The shown snapshot corresponds to $t=300 \mathrm{~s}$ and shows the formation of an expanding dispersive shock.

Linearized dispersion. Since the SGN regularizing term in the energy is nonlinear in $h$, it is of interest to check whether the emergence of the shock-like stationary front is conditioned by this 

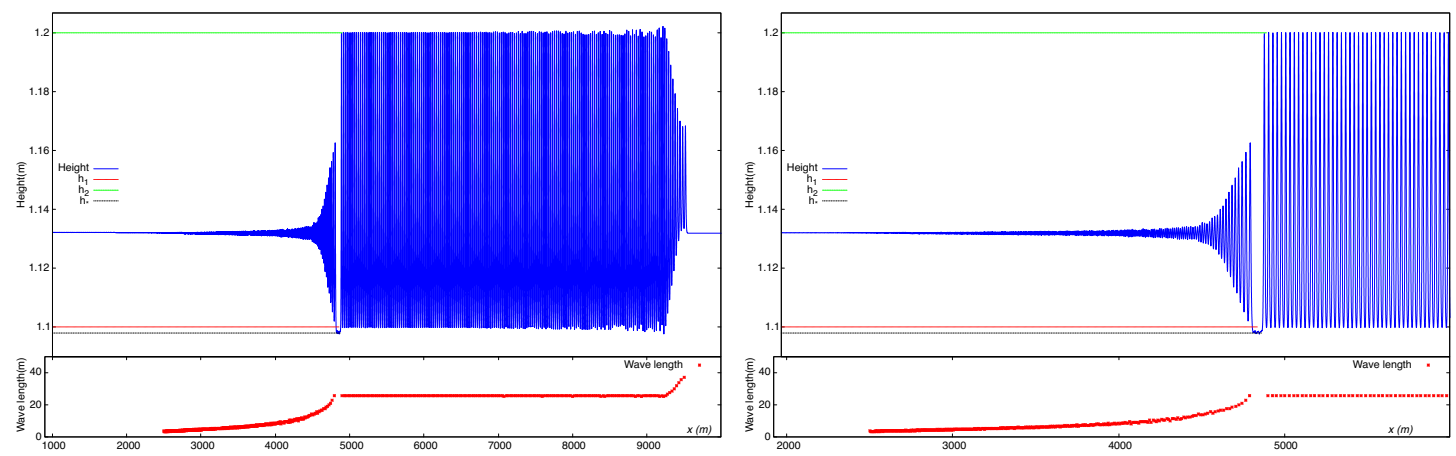

Figure 13: Numerical solution of the generalized Riemann problem for the Boussinesq system; the corresponding pressure is given by (53). The snapshot at $t=1000 \mathrm{~s}$ (left figure) shows the formation of a stationary shock which is similar to the one obtained for the SGN model in Figure 8. A zoom in on the transition front is shown on the right figure.

(non-fundamental) nonlinearity. To this end, we now consider the Boussinesq type approximation of the SGN system where the dispersive term in the energy is linearized. We obtain the same system of equations (30) where now

$$
p=\frac{g h^{2}}{2}+\frac{\bar{h}_{0}^{2}}{3} h_{t t} .
$$

In contrast to the original problem, the coefficient $\bar{h}_{0}$ in (53) is constant. The corresponding TW solutions satisfy the equations:

$$
h(u-D)=m, \frac{m^{2}}{h}+\frac{g}{2} h^{2}+\frac{D^{2} \bar{h}_{0}^{2}}{3} h^{\prime \prime}=\iota .
$$

where, again, $m$ and $\iota$ are integration constants. Multiplying the second equation by $h^{\prime}$ and integrating once we obtain:

$$
\frac{D^{2} \bar{h}_{0}^{2}}{6}\left(h^{\prime}\right)^{2}=\iota h-\frac{g h^{3}}{6}-m^{2} \ln \left(\frac{h}{H}\right) .
$$

Here $H$ is a new positive integration constant. The three real positive roots $0<h_{0}<h_{1}<h_{2}$ of the right-hand side of (54) exist if the equation $\frac{m^{2}}{h}+\frac{g}{2} h^{2}=\iota$ has two positive roots; then the solution oscillates between the values $h_{1}$ and $h_{2}$.

If we choose $\iota \approx 19.22372308, m^{2} \approx 14.4889747, H \approx 0.297886$, we obtain the same roots as in the case of the periodic solution considered in the SGN setting: $h_{0} \approx 1.0962, h_{1} \approx 1.1$ and $h_{2} \approx 1.2$. The corresponding wave length is :

$$
\lambda=\frac{2 D \bar{h}_{0}}{\sqrt{6}} \int_{h_{1}}^{h_{2}}\left(\iota h-\frac{g h^{3}}{6}-m^{2} \ln \left(\frac{h}{H}\right)\right)^{-1 / 2} d h .
$$

We can now prescribe the same value of $D$ as in the generalized Riemann problem for the SGN system, and take $\bar{h}_{0}=h_{0}$. The main difference between SGN and Boussinesq models is that the latter is not invariant under the Galilean transformation which implies that the wave length in the Boussinesq model depends on the phase velocity $D$. 
The numerical solution of the Boussinesq model with the same initial data as in the SGN model (Test 1) is illustrated in Fig. 13. Once again we see the formation of a stationary transition front separating 'cold' and 'hot' states which points to the robustness of our results.

\section{Conclusions}

Hyperbolic conservation laws are ubiquitous in continuum physics. They describe adequately the dynamics of dissipation-free systems at large scales where the fields of interest are sufficiently smooth. The associated models, however, fail to adequately represent the underlying physics at small scales which emerges inevitably due to the inherent nonlinearity.

The localization trend in hyperbolic systems leads to the formation of concentrations and/or oscillations. To describe the underlying microscale phenomena in some detail, the hyperbolic system needs to be regularized dispersively which leads to the appearance in the model of the characteristic length and time scales.

In this paper we studied a class of temporarily regularized dispersive models which we showed to generate highly localized and apparently stationary transition fronts. The existence of such kinktype solutions in a model with convex energy is rather remarkable. More interestingly, if formally averaged, such macroscopic discontinuities become unstable hyperbolic shocks. The evidence of stability for these solutions in the dispersive setting was obtained so far only numerically: our simulations strongly suggest that they have at least a finite reserve of stability and that they are long-living. More analytical work is needed to see if these shock-like fronts are indeed stable, at least in the zero dispersion limit when they degenerate into jump discontinuities. The adequate averaging of such solutions must necessarily involve the account of degrees of freedom associated with micro-oscillations as in Whitham's averaging method.

In the case of SGN system, the Whitham's approach can be shown to produce a hyperbolic problem [60]. The corresponding higher dimensional system generates singularities and we conjecture that at least some of these singularities can be interpreted as the limits of the dispersive shock-like fronts studied in this paper. An important step along these lines was made in [57] where the discontinuities emerging in the zero dispersion limit of the fifth order KdV were shown to be stable shock solutions of the corresponding Whitham equations. In [57] the shocks in Whitham's equations were shown to emerge as pointwise limits of the smooth TW solutions of dispersive equations representing heteroclinic connections between two periodic orbits of an ODE. The dispersion considered in this paper is too 'poor' to support such smooth 'homogeneous-to-periodic' heteroclinic TW solutions. In this situation we expect the limiting Whitham shocks to result from the non-smooth solutions of the corresponding ODEs.

Acknowledgments. The authors thank the anonymous referees for helpful suggestions. SG was supported by the grants ANR-11-LABEX-0092 and ANR-11-IDEX-0001-02). BN was supported by the Inria-MOST Associate team program AMoSS and by the grant MOST-105-2911I-006-502. KMS was partially supported by the grant MOST 105-2115-M-002-013-MY2. LT was supported by the grant ANR-10-IDEX-0001-02.

\section{A Lagrangian and Eulerian descriptions}

Since our general theory is formulated in Lagrangian coordinates, which are usually used in solid mechanics, while the analysis of the SGN model, which originates in fluid mechanics, is performed 
in Eulerian description, we briefly recall here the connection between the two ways of describing the motion of a $1 \mathrm{D}$ continuum $[52,55]$.

If $t$ is time, and $q$ is the (mass) Lagrangian coordinate, the spatial Eulerian coordinate is defined through the motion of the continuum $x=x(t, q)$. The mass conservation equation in the Lagrangian coordinates can be written as $\rho(t, q) x_{q}=\rho_{0}(q)$, where $\rho(t, q)$ and $\rho_{0}(q)$ are the actual and the reference mass densities, so that $d m=\rho_{0}(q) d q=\rho(t, q) d x$. One can see that if we choose $\rho_{0}(q) \equiv 1$, the Lagrangian variable $q$ will effectively coincides with the mass $m$; in this case the mass balance equation will take a particularly simple form $x_{q} \equiv w=\rho^{-1}$ [52], [55]. Making this the definition for the specific volume (the strain) $w$ and defining the fluid velocity $u$ as $u \equiv x_{t}$ we obtain $d x=u d t+w d q$. Note that the partial derivative with respect to $t$ in such Lagrangian coordinates $(t, q)$ becomes the material derivative in the Eulerian coordinates $(t, x)$ and we can write $f_{t}(t, q)=\frac{d f(t, x)}{d t}=f_{t}(t, x)+u(t, x) f_{x}(t, x)$. A general conservation law in the Lagrangian coordinates $a_{t}+b_{q}=0$, can be rewritten in the Eulerian coordinates as $(\rho a)_{t}+(\rho u a+b)_{x}=0$. Indeed, consider a closed contour $C_{0}$ in $(t, q)$ space. Then, the conservation law can be written in the integral form as $\oint_{C_{0}}-a d q+b d t=0$. In Eulerian coordinates this integral transforms into $\oint_{C_{t}}-\frac{a}{w} d x+\left(\frac{a u}{w}+b\right) d t=0$, where $C_{t}$ is the image of $C_{0}$ in $(t, x)$ space. These integral relations can be also used to relate jump conditions in the two spaces. Consider a shock having the velocity $V$ in Lagrangian coordinates. The corresponding Rankine - Hugoniot relation reads $-V[a]+[b]=0$. The same shock will have the velocity $V^{E}$ in Eulerian coordinates and $-V=\rho\left(u-V^{E}\right)$. In terms of $V^{E}$ the above Rankine - Hugoniot relation takes the form $\left[\rho\left(u-V^{E}\right) a\right]+[b]=0$.

\section{B Sonic wave speeds}

Here we show the inequalities (45), (46) and (47) are always satisfied for the jumps moving to the right $(m<0)$. If $m>0$, some obvious changes in the signs of the inequalities should be done. Suppose that $m<0$ and consider the polynomial (44) :

$$
Q\left(h_{\star}\right)=h_{\star}^{3}-\left(h_{0} h_{1}+h_{0} h_{2}+h_{1} h_{2}\right) h_{\star}+2 h_{0} h_{1} h_{2}=\left(h_{\star}-h_{\star}^{0}\right)\left(h_{\star}-h_{\star}^{-}\right)\left(h_{\star}-h_{\star}^{+}\right) .
$$

Since $Q(0)>0$, there exists a negative root $h_{\star}^{0}$. Since $Q\left(h_{0}\right)>0$ and $Q\left(h_{1}\right)<0$, there exists a positive root $h_{\star}^{-}$between $h_{0}$ and $h_{1}$. Since $Q\left(h_{1}\right)<0$ and $Q\left(h_{2}\right)>0$, there exists a positive root $h_{\star}^{+}$between $h_{1}$ and $h_{2}$. Hence,

$$
h_{\star}^{0}<0<h_{0}<h_{\star}^{-}<h_{1}<h_{\star}^{+}<h_{2},
$$

and the inequalities (45) are established. Vieta's formulas for $Q\left(h_{\star}\right)$ are :

$$
\begin{gathered}
2 h_{0} h_{1} h_{2}=-h_{\star}^{0} h_{\star}^{-} h_{\star}^{+}, \quad h_{0} h_{1}+h_{0} h_{2}+h_{1} h_{2}=h_{\star}^{-} h_{\star}^{+}+h_{\star}^{0} h_{\star}^{-}+h_{\star}^{0} h_{\star}^{+}, \\
h_{\star}^{0}+h_{\star}^{-}+h_{\star}^{+}=0
\end{gathered}
$$

Using the expression for $m^{2}=g h_{0} h_{1} h_{2}$, the mass conservation law and Vieta's formulas written above, one obtains

$$
u_{\star}^{-}+\sqrt{g h_{\star}^{-}}=\frac{m}{h_{\star}^{-}}+\sqrt{g h_{\star}^{-}}=\frac{-\sqrt{g h_{0} h_{1} h_{2}}}{h_{\star}^{-}}+\sqrt{g h_{\star}^{-}}
$$




$$
\begin{gathered}
=\frac{-\sqrt{\frac{-g h_{\star}^{0} h_{\star}^{-} h_{\star}^{+}}{2}}}{h_{\star}^{-}}+\sqrt{g h_{\star}^{-}} \\
=-\sqrt{\frac{g\left(h_{\star}^{-}+h_{\star}^{+}\right) h_{\star}^{+}}{2 h_{\star}^{-}}}+\sqrt{g h_{\star}^{-}} \\
<-\sqrt{\frac{g\left(h_{\star}^{-}+h_{\star}^{+}\right)}{2}}+\sqrt{g h_{\star}^{-}}<0
\end{gathered}
$$

because $h_{\star}^{+}>h_{\star}^{-}$. The inequality $u_{\star}^{+}+\sqrt{g h_{\star}^{+}}>0$ can be proven in the same way. The inequalities $u_{\star}^{+}-\sqrt{g h_{\star}^{+}}<0$ and $u_{\star}^{+}-\sqrt{g h_{\star}^{+}}<0$ are trivial because for the right facing stationary waves the velocities $u_{\star}^{ \pm}$are negative.

\section{Numerical method}

Overview. To find approximate solutions to SGN equations, we used the hyperbolic-elliptic splitting approach developed previously in [39]. Our modified version of this algorithm will be presented in the form of two steps:

Hyperbolic step. At each time step $\Delta t$ we solve the hyperbolic part of the system (30) written in the form :

$$
\boldsymbol{q}_{t}+\boldsymbol{f}(\boldsymbol{q}, u)_{x}=\boldsymbol{\psi}(\boldsymbol{q}, u)
$$

where $\boldsymbol{q}=(h, h K)^{T}, \boldsymbol{f}=\left(h u, h K u+\frac{1}{2} g h^{2}\right)^{T}, K=u-\frac{1}{3 h}\left(h^{3} u_{x}\right)_{x}$, and $\boldsymbol{\psi}=\left(0,\left(\frac{2}{3} h^{3}\left(u_{x}\right)^{2}\right)_{x}\right)^{T}$.

Elliptic step. Using the approximate solutions $h$ and $K$ computed during the hyperbolic step, we invert numerically the elliptic operator:

$$
u-\frac{1}{3 h}\left(h^{3} u_{x}\right)_{x}=K
$$

with the prescribed boundary conditions.

Note that on the hyperbolic step, rather than writing (56a) in the conservation form as in [39] with $\boldsymbol{f}=\left(h u, h K u+\frac{1}{2} g h^{2}-\frac{2}{3} h^{3}\left(u_{x}\right)^{2}\right)^{T}$ and $\boldsymbol{\psi}=\mathbf{0}$ (which is ideal in the conservative first-order setting [40], but is difficult to make higher than first order accurate), we write it in the form of a balance law. We then obtain a standard elliptic problem which any state-of-the-art method can resolve $[41,58]$.

Detailed numerical method for the hyperbolic step. To compute solutions to SGN equations in the hyperbolic step, we use the semi-discrete finite volume method written in a wavepropagation form (cf. [34, 35]). This method belongs to the class of flux-vector splitting methods for hyperbolic conservation laws [25, 40,61], and has been applied to compressible multiphase flows (cf. [54]), and in other instances of practical importance. For simplicity, we describe the method on a uniform grid of $N$ cells with fixed mesh spacing $\Delta x$. The method is based on a staggered grid formulation in which the value $\boldsymbol{Q}_{j}(t)$ approximates the cell average of the solutions $\boldsymbol{q}$ over the grid cell $C_{j}$ : 


$$
\boldsymbol{Q}_{j}(t) \approx \frac{1}{\Delta x} \int_{x_{j-1 / 2}}^{x_{j+1 / 2}} \boldsymbol{q}(t, x) d x
$$

while $U_{j}(t) \approx u\left(t, x_{j}\right)$ gives the pointwise approximation of the velocity $u$ at $x_{j}$ at time $t$.

The semi-discrete version of the wave-propagation method is a method-of-lines discretization of (56a) that can be written as a system of ordinary differential equations (ODEs) in the form :

$$
\frac{d \boldsymbol{Q}_{j}}{d t}=\mathcal{L}_{j}(\boldsymbol{Q}, U)
$$

with

$$
\mathcal{L}_{j}(\boldsymbol{Q}, U)=-\frac{1}{\Delta x}\left(\mathcal{A}^{+} \Delta \boldsymbol{Q}_{j-1 / 2}+\mathcal{A}^{-} \Delta \boldsymbol{Q}_{j+1 / 2}+\mathcal{A} \Delta \boldsymbol{Q}_{j}\right)+\boldsymbol{\Psi}_{j}(\boldsymbol{Q}, U)
$$

for $j=1,2, \ldots, N$. Here, $\boldsymbol{Q}$ and $U$ are the vectors with components $\boldsymbol{Q}_{j}$ and $U_{j}$ respectively, $\mathcal{A}^{+} \Delta \boldsymbol{Q}_{j-1 / 2}$ and $\mathcal{A}^{-} \Delta \boldsymbol{Q}_{j+1 / 2}$, are the right- and left-moving fluctuations, respectively, that are entering into the grid cell $C_{j}$, and $\mathcal{A} \Delta \boldsymbol{Q}_{j}$ is the total fluctuation within the cell. To determine these fluctuations, we need to solve Riemann problems (see below). Note that the term $\boldsymbol{\Psi}_{j}(\boldsymbol{Q}, U)$ in (57b) represents a discrete version of $\boldsymbol{\psi}$ over the grid cell $C_{j}$ which can be evaluated straightforwardly by numerical differentiation techniques such as the finite-difference approximation of derivatives (cf. [41]).

Consider now the fluctuations $\mathcal{A}^{ \pm} \Delta \boldsymbol{Q}_{j-1 / 2}$ arising from the edge $(j-1 / 2)$ between cells $C_{j-1}$ and $C_{j}$, for example. This amounts to solving the Cauchy problem for the homogeneous part of (56a) in the form :

$$
\begin{cases}\boldsymbol{q}_{t}+\boldsymbol{f}\left(\boldsymbol{q}, u_{j-1 / 2}^{L}\right)_{x}=0 & \text { if } x<x_{j-1 / 2} \\ \boldsymbol{q}_{t}+\boldsymbol{f}\left(\boldsymbol{q}, u_{j-1 / 2}^{R}\right)_{x}=0 & \text { if } x>x_{j-1 / 2}\end{cases}
$$

with the piecewise constant initial data at a given time $t_{0}$ :

$$
\boldsymbol{q}\left(t_{0}, x\right)= \begin{cases}\boldsymbol{q}_{j-1 / 2}^{L} & \text { if } x<x_{j-1 / 2} \\ \boldsymbol{q}_{j-1 / 2}^{R} & \text { if } x>x_{j-1 / 2}\end{cases}
$$

Here $\boldsymbol{q}_{j-1 / 2}^{L}=\lim _{x \rightarrow x_{(j-1 / 2)}} \tilde{\boldsymbol{q}}_{j-1}(x)$ and $\boldsymbol{q}_{j-1 / 2}^{R}=\lim _{x \rightarrow x_{(j-1 / 2)}} \tilde{\boldsymbol{q}}_{j}(x)$ are the interpolated states obtained by taking limits of the reconstructed piecewise-continuous function $\tilde{\boldsymbol{q}}_{j-1}(x)$ or $\tilde{\boldsymbol{q}}_{j}(x)$ (each of them can be determined by applying a standard interpolation scheme to the set of discrete data $\left\{\boldsymbol{Q}_{j}\left(t_{0}\right)\right\}$, see $[25,40,53]$ for more details) to the left and right of the cell edge at $x_{j-1 / 2}$, respectively. To find the set of interpolate states of $\left\{u_{j-1 / 2}^{L}\right\}$ and $\left\{u_{j-1 / 2}^{R}\right\}$, the approach we propose here is to solve the elliptic equation (56b) based on the the sets of data $\left\{\boldsymbol{q}_{j-1 / 2}^{L}\right\}$ and $\left\{\boldsymbol{q}_{j-1 / 2}^{R}\right\}$, respectively, which is a consistent approximation of $u$ in the SGN model at the cell edges.

Note that if the conservative version of the flux $\boldsymbol{f}$ is being used in the problem formulation [39], the governing equation in the Riemann problem would be

$$
\begin{cases}\boldsymbol{q}_{t}+\boldsymbol{f}\left(\boldsymbol{q}, u_{j-1 / 2}^{L},\left(u_{x}\right)_{j-1 / 2}^{L}\right)_{x}=0 & \text { if } x<x_{j-1 / 2}, \\ \boldsymbol{q}_{t}+\boldsymbol{f}\left(\boldsymbol{q}, u_{j-1 / 2}^{R},\left(u_{x}\right)_{j-1 / 2}^{R}\right)_{x}=0 & \text { if } x>x_{j-1 / 2} .\end{cases}
$$


Then it should be clear that the need to interpolate the set of states $\left\{\left(u_{x}\right)_{j-1 / 2}^{L}\right\}$ and $\left\{\left(u_{x}\right)_{j-1 / 2}^{R}\right\}$ consistently and to be more than first-order accurate would complicate the matter further, and so it is preferable to use (56a) as the basis in the hyperbolic part of the method.

Here we are interested in the HLL (Harten, Lax, and van Leer) approximate solver [29] for the numerical resolution of the Riemann problem (58) where the basic structure of the solution is assumed to be composed of two discontinuities propagating at constant speeds $s_{j-1 / 2}^{L}$ and $s_{j-1 / 2}^{R}$ to the left and right, $s_{j-1 / 2}^{L}<s_{j-1 / 2}^{R}$, separating three constant states in the space-time domain. We assume that $s_{j-1 / 2}^{L}$ and $s_{j-1 / 2}^{R}$ are known a priori by some simple estimates based on the local information of the wave speeds (cf. [61,39]). Then it is easy to find the constant state in the middle region, denoted by $\boldsymbol{q}_{j-1 / 2}^{*}$, as

$$
\boldsymbol{q}_{j-1 / 2}^{*}=\frac{s_{j-1 / 2}^{R} \boldsymbol{q}_{j-1 / 2}^{R}-s_{j-1 / 2}^{L} \boldsymbol{q}_{j-1 / 2}^{L}-\boldsymbol{f}\left(\boldsymbol{q}_{j-1 / 2}^{R}, u_{j-1 / 2}^{R}\right)+\boldsymbol{f}\left(\boldsymbol{q}_{j-1 / 2}^{L}, u_{j-1 / 2}^{R}\right)}{s_{j-1 / 2}^{R}-s_{j-1 / 2}^{L}},
$$

see [61] for more details. We then find the expression for the fluctuations in terms of jumps across each discontinuity :

$$
\mathcal{A}^{ \pm} \Delta \boldsymbol{Q}_{j-1 / 2}=\left(s_{j-1 / 2}^{L}\right)^{ \pm}\left(\boldsymbol{q}_{j-1 / 2}^{*}-\boldsymbol{q}_{j-1 / 2}^{L}\right)+\left(s_{j-1 / 2}^{R}\right)^{ \pm}\left(\boldsymbol{q}_{j-1 / 2}^{R}-\boldsymbol{q}_{j-1 / 2}^{*}\right),
$$

where $s^{+}=\max (s, 0)$ and $s^{-}=\min (s, 0)$.

Similarly, we can define fluctuation $\mathcal{A} \Delta \boldsymbol{Q}_{j}$ within cell $C_{j}$ based on the solution of the following Riemann problem at the cell center $x_{j}$ :

$$
\begin{cases}\boldsymbol{q}_{t}+\boldsymbol{f}\left(\boldsymbol{q}, u_{j-1 / 2}^{R}\right)_{x}=0 & \text { if } x<x_{j}, \\ \boldsymbol{q}_{t}+\boldsymbol{f}\left(\boldsymbol{q}, u_{j+1 / 2}^{L}\right)_{x}=0 & \text { if } x>x_{j}\end{cases}
$$

with the initial condition

$$
\boldsymbol{q}\left(t_{0}, x\right)= \begin{cases}\boldsymbol{q}_{j-1 / 2}^{R} & \text { if } x<x_{j}, \\ \boldsymbol{q}_{j+1 / 2}^{L} & \text { if } x>x_{j} .\end{cases}
$$

To integrate the system of ODEs (57a) in time, we employ the strong stability-preserving (SSP) multistage Runge-Kutta scheme [28]. That is, in the first-order case we use the Euler forward time discretization as

$$
\boldsymbol{Q}_{j}^{n+1}=\boldsymbol{Q}_{j}^{n}+\Delta t \mathcal{L}_{j}\left(\boldsymbol{Q}^{n}, U^{n}\right),
$$

where we start with the cell average $\boldsymbol{Q}_{j}^{n} \approx \boldsymbol{Q}_{j}\left(t_{n}\right)$ and $U^{n} \approx U\left(t_{n}\right)$ at time $t_{n}$, yielding the solution at the next time step $\boldsymbol{Q}_{j}^{n+1}$ over $\Delta t=t_{n+1}-t_{n}$. In the second-order case, however, we use the classical two-stage Heun method (also called the modified Euler method) as :

$$
\begin{aligned}
\boldsymbol{Q}_{j}^{*} & =\boldsymbol{Q}_{j}^{n}+\Delta t \mathcal{L}_{j}\left(\boldsymbol{Q}^{n}, U^{n}\right), \\
\boldsymbol{Q}_{j}^{n+1} & =\frac{1}{2} \boldsymbol{Q}_{j}^{n}+\frac{1}{2} \boldsymbol{Q}_{j}^{*}+\frac{1}{2} \Delta t \mathcal{L}_{j}\left(\boldsymbol{Q}^{*}, U^{*}\right) .
\end{aligned}
$$


It is common that the three-stage third-order scheme of the form

$$
\begin{aligned}
\boldsymbol{Q}_{j}^{*} & =\boldsymbol{Q}_{j}^{n}+\Delta t \mathcal{L}_{j}\left(\boldsymbol{Q}^{n}, U^{n}\right) \\
\boldsymbol{Q}_{j}^{* *} & =\frac{3}{4} \boldsymbol{Q}_{j}^{n}+\frac{1}{4} \boldsymbol{Q}_{j}^{*}+\frac{1}{4} \Delta t \mathcal{L}_{j}\left(\boldsymbol{Q}^{*}, U^{*}\right), \\
\boldsymbol{Q}_{j}^{n+1} & =\frac{1}{3} \boldsymbol{Q}_{j}^{n}+\frac{2}{3} \boldsymbol{Q}_{j}^{*}+\frac{2}{3} \Delta t \mathcal{L}_{j}\left(\boldsymbol{Q}^{* *}, U^{* *}\right) .
\end{aligned}
$$

is a preferred one to be used in conjunction with the third- or fifth-order WENO (weighted essentially non-oscillatory) scheme that is employed for the reconstruction of $\tilde{\mathbf{q}}_{j}(x)$ during the spatial discretization (cf. [53]).

Detailed numerical method for the elliptic step. To find the flow velocity $u$ in SGN model at a given time $t$, the elliptic equation (56b) is solved with $h$ and $K$ known a priori, and subject to the prescribed boundary conditions (such as the Neumann and periodic boundaries considered here) at both ends. For simplicity, we use a three-point finite difference method on a uniform grid with mesh spacing $\Delta x$ by first taking a backward difference for the outer derivative and then a forward difference for the inner derivative; collecting terms, we get the following constant coefficient difference formula for node $j$ :

$$
\alpha_{j} U_{j-1}+\beta_{j} U_{j}+\gamma_{j} U_{j-1}=K_{j},
$$

with $\alpha_{j}, \beta_{j}$, and $\gamma_{j}$ defined by

$$
\begin{aligned}
\alpha_{j} & =-\frac{1}{3 H_{j}} \frac{\left(H^{3}\right)_{j-1 / 2}}{(\Delta x)^{2}}, \\
\beta_{j} & =\frac{1}{3 H_{j}}\left(\frac{\left(H^{3}\right)_{j-1 / 2}}{(\Delta x)^{2}}+\frac{\left(H^{3}\right)_{j+1 / 2}}{(\Delta x)^{2}}\right)+1, \\
\gamma_{j} & =-\frac{1}{3 H_{j}} \frac{\left(H^{3}\right)_{j+1 / 2}}{(\Delta x)^{2}}
\end{aligned}
$$

respectively, where $\left(H^{3}\right)_{j \pm 1 / 2}=\left(\left(H_{j}\right)^{3}+\left(H_{j \pm 1}\right)^{3}\right) / 2 \approx\left(h\left(x_{j \pm 1 / 2}, t\right)\right)^{3}$ (cf. [41]). Going through all the nodal points for $j=1,2, \cdots, N$, and using the boundary conditions, we obtain a nonsingular linear system for the unknown velocity $U(t)$.

Let $\tau_{j}$ be the local truncation error of (61) to the elliptic equation (56b), i.e.,

$$
\tau_{j}=\tilde{\alpha}_{j} u\left(t, x_{j-1}\right)+\tilde{\beta}_{j} u\left(t, x_{j}\right)+\tilde{\gamma}_{j} u\left(t, x_{j-1}\right)-K\left(t, x_{j}\right),
$$

where

$$
\begin{aligned}
& \tilde{\alpha}_{j}=-\frac{1}{3 h\left(t, x_{j}\right)} \frac{h^{3}\left(t, x_{j-1 / 2}\right)}{(\Delta x)^{2}}, \\
& \tilde{\beta}_{j}=\frac{1}{3 h\left(t, x_{j}\right)}\left(\frac{h^{3}\left(t, x_{j-1 / 2}\right)}{(\Delta x)^{2}}+\frac{h^{3}\left(t, x_{j+1 / 2}\right)}{(\Delta x)^{2}}\right)+1, \\
& \tilde{\gamma}_{j}=-\frac{1}{3 h\left(t, x_{j}\right)} \frac{h^{3}\left(t, x_{j+1 / 2}\right)}{(\Delta x)^{2}} .
\end{aligned}
$$


Then it is easy to show that $\tau_{j}$ is on the order of $(\Delta x)^{2}$, i.e.,

$$
\tau_{j}=-\frac{(\Delta x)^{2}}{12 h\left(t, x_{j}\right)}\left(\frac{1}{3} h^{3}\left(t, x_{j}\right) u_{x x x x}\left(t, x_{j}\right)+\frac{2}{3} h_{x}^{3}\left(t, x_{j}\right) u_{x x x}\left(t, x_{j}\right)\right)+O\left((\Delta x)^{4}\right),
$$

and hence (61) is a second-order approximation to (56b) locally; the second-order global error of the method can be ensured, when the method remains stable, i.e., the inverse of the matrix of the resulting linear system from the finite-difference approximation can be bounded by some constant independent of $\Delta x$, as $\Delta x \rightarrow 0$ (cf. [41]).

Validation tests. To access the numerical accuracy of our method, we performed convergence studies for the two benchmark tests where the exact solutions are readily available for comparison. In all the tests, the gravitational constant was chosen to be $g=10 \mathrm{~m} / \mathrm{s}^{2}$, and the Courant number was set to 0.5 to ensure the stability of the hyperbolic solver.

Our first test is the propagation of a single solitary wave in a fluid which is at rest at infinity, see (39). We set $h_{1}=10 \mathrm{~m}$ and $h_{2}=22.5 \mathrm{~m}$, yielding $D=15 \mathrm{~m} / \mathrm{s}$; the computational domain was of size $300 m$ with periodic boundary conditions at both ends.

Our Table 1 shows 1-norm errors of the height at time $t=40 \mathrm{~s}$ (time it takes the solitary wave crest to travel one period) for a convergence study of the solutions obtained using our numerical strategy with four different mesh sizes $N=1200,2400,4800$, and 9600, and three different hyperbolic integration schemes. The underlying elliptic solver for (61) is the second-order finite difference scheme.

Let $E^{1}(h)=\left\{E_{j}^{1}(h)\right\}$ for $j=1,2,3,4$ be the sequence of the 1-norm error of the computed height $h$ to its true solution on an $N=\{1200,2400,4800,9600\}$ grid. With that, it is a common practice to estimate the rate of convergence using the errors on two consecutive grids based on the formula

$$
\text { convergence order }=\frac{\ln \left(E_{j-1}^{1}(h) / E_{j}^{1}(h)\right)}{\ln \left(N_{j-1} / N_{j}\right)} .
$$

From Table 1, we observe that when Godunov method is employed in the hyperbolic step, (i.e., the method uses zeroth-order piecewise constant reconstruction scheme for the Riemann data at the cell edges, and the forward Euler method (60a) for the time discretization), the order of accuracy of algorithm approaches to first-order accurate as the mesh is refined, and it is secondorder accurate, when MUSCL (monotonic upstream-centered scheme for conservation laws) is employed alternatively (i.e., both the first-order piecewise linear reconstruction scheme and the Heun method (60b) are in use). In the WENO 3 case, however, (i.e., the method uses the third-order WENO (weighted essentially non-oscillatory) scheme for Riemann data reconstruction, and the third-order method (60c) for the time discretization), the order of accuracy in average is 2.1 approximately which is less than 3 (the formal order of accuracy of the hyperbolic solver WENO 3); this result may not come as a surprise because our underlying elliptic solver is only of $O\left((\Delta x)^{2}\right)$. Nevertheless, among all the three methods, WENO 3 gives the smallest error in magnitude for each mesh size.

Our second example concerns the propagation of a $T W$ in a periodic domain of one wave length, see (37).

The periodic wave parameters were chosen to be $h_{0} \approx 1.0962 \mathrm{~m}, h_{1}=1.1 \mathrm{~m}$, and $h_{2}=1.2 \mathrm{~m}$. This yields the wave speed $D \approx 3.36413 \mathrm{~m} / \mathrm{s}$ and wave length $\lambda \approx 26.3767 \mathrm{~m}$. The computational domain was taken of the size of one wave length with periodic boundary conditions at both ends. 
Table 1: Numerical results for the solitary wave problem obtained using our algorithm with four different mesh sizes and three different hyperbolic integration schemes; 1-norm errors in the height are shown at time $t=40 \mathrm{~s}$. The elliptic equation (61) is solved using second-order finite difference scheme in all cases.

\begin{tabular}{|c|cc|cc|cc|}
\hline Hyperbolic step & \multicolumn{2}{|c|}{ Godunov } & \multicolumn{2}{c|}{ MUSCL } & \multicolumn{2}{c|}{ WENO 3 } \\
\hline$N$ & $E^{1}(h)$ & order & $E^{1}(h)$ & order & $E^{1}(h)$ & order \\
\hline 1200 & $2.595 \mathrm{e}+02$ & & $4.894 \mathrm{e}+00$ & & $2.622 \mathrm{e}-01$ & \\
2400 & $1.470 \mathrm{e}+02$ & 0.82 & $1.210 \mathrm{e}+00$ & 2.02 & $4.410 \mathrm{e}-02$ & 2.57 \\
4800 & $7.834 \mathrm{e}+01$ & 0.91 & $3.005 \mathrm{e}-01$ & 2.01 & $1.178 \mathrm{e}-02$ & 1.90 \\
9600 & $4.044 \mathrm{e}+01$ & 0.95 & $7.487 \mathrm{e}-02$ & 2.01 & $3.060 \mathrm{e}-03$ & 1.94 \\
\hline
\end{tabular}

Table 2: Numerical results for the periodic TW problem; 1-norm errors in the height are shown at the time where the wave travelled over four periodic distance of the domain.

\begin{tabular}{|c|cc|cc|cc|}
\hline Hyperbolic step & \multicolumn{2}{|c|}{ Godunov } & \multicolumn{2}{c|}{ MUSCL } & \multicolumn{2}{c|}{ WENO 3 } \\
\hline$N$ & $E^{1}(h)$ & order & $E^{1}(h)$ & order & $E^{1}(h)$ & order \\
\hline 300 & $1.346 \mathrm{e}-01$ & & $5.250 \mathrm{e}-03$ & & $3.521 \mathrm{e}-03$ & \\
600 & $7.749 \mathrm{e}-02$ & 0.83 & $1.094 \mathrm{e}-03$ & 2.37 & $4.563 \mathrm{e}-04$ & 3.09 \\
1200 & $4.100 \mathrm{e}-02$ & 0.92 & $2.482 \mathrm{e}-04$ & 2.15 & $5.927 \mathrm{e}-05$ & 2.96 \\
2400 & $2.112 \mathrm{e}-02$ & 0.96 & $6.072 \mathrm{e}-05$ & 2.03 & $7.923 \mathrm{e}-06$ & 2.90 \\
\hline
\end{tabular}

The results of the convergence study in this case are shown in Table 2. From the table, we observe similar rate of convergence as in Table 1, when the Godunov and the MUSCL methods are in used in the hyperbolic step of the algorithm, and a slightly better behavior of error when WENO 3 is employed.

Finally, we present numerical results for the simulation of a dam break problem studied, for instance, in $[12,39,48]$. Since there is no analytical solution to this problem, such a study is rather qualitative, but it allows us to recover some qualitative characteristics of the solution (the amplitude of the leading wave and its velocity, for example). We take the velocity vanishing in the entire computational domain of size $x \in[-300,300] \mathrm{m}, u(0, x)=0 \mathrm{~m} / \mathrm{s}$, while the water depth is piece-wise constant :

$$
h(0, x)= \begin{cases}h_{L}, & \text { if } x<0, \\ h_{R}, & \text { if } x \geq 0,\end{cases}
$$

where $h_{L}$ and $h_{R}$ are chosen to be $1.8 \mathrm{~m}$ and $1 \mathrm{~m}$, respectively. The discontinuous initial data for the water depth will be replaced by a smooth function :

$$
h(0, x)=h_{R}+\frac{h_{L}-h_{R}}{2}\left(1-\tanh \left(\frac{x}{\alpha}\right)\right),
$$

where $\alpha=2 m$ or $\alpha=0.4 \mathrm{~m}$. The structure of the solution (but not the velocity of the leading solitary wave and its velocity) depends on the value of $\alpha$. According to the terminology given in [48], the case $\alpha=2 \mathrm{~m}$ produces $S_{2}$ configuration (flat structure of the fluid depth behind the 

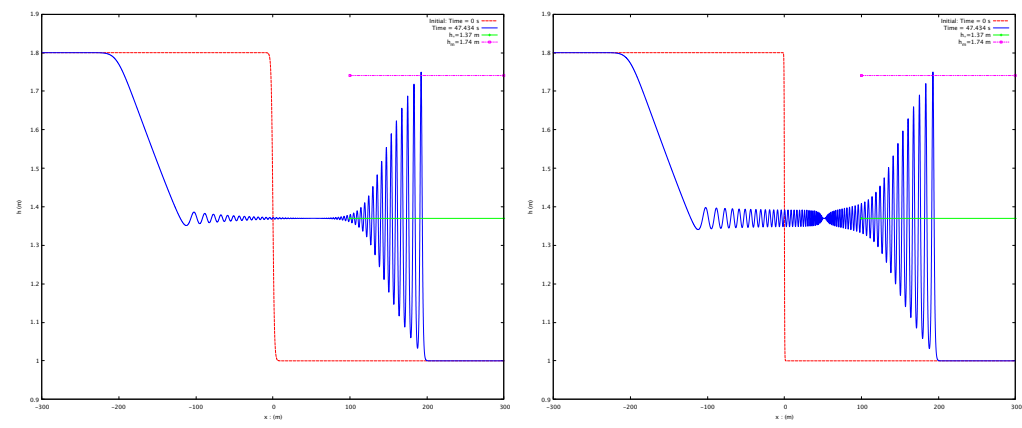

Figure 14: Left figure : Numerical result for the dam break problem for the initial data (63) with $\alpha=2 m\left(S_{2}\right.$ case in the terminology of [48]). The solid line is the water depth at time $t=47.434 \mathrm{~s}$, and the dashed line is the initial condition. The lines for $h_{*}$ and $h_{m}$ are the depths of the post rightgoing undular bore and the leading solitary wave (cf. [12]), respectively. Right figure : Numerical result for the dam break problem for the initial data (63) with $\alpha=0.4 \mathrm{~m}\left(S_{3}\right.$ case in the terminology of [48]). The solid line is the water depth at time $t=47.434 \mathrm{~s}$, and the dashed line is the initial condition. The lines for $h_{*}$ and $h_{m}$ are the depths of the post right-going undular bore and the leading solitary wave (cf. [12]), respectively.

dispersive shock, Figure 14, (left), while $\alpha=0.4 \mathrm{~m}$ produces $S_{3}$ configuration (existence of a node type point in the fluid depth profile, Figure 14, (right). The node point moves with the velocity which can be estimated by using the continuity through dispersive shock of the Riemann invariant of the corresponding Saint-Venant equations describing the waves advancing to the right.

The comparison of the analytical and numerical results for the amplitude of the leading solitary wave is shown in Fig. 14 at time $t=47.434 s$ with the mesh size $\Delta x=0.025 m$ (i.e., $N=24000$ meshes). As far as the global wave structure is concerned, our results are in good agreement with the ones shown in [12] at time $t=150 \mathrm{~s}$, where a different value of the gravitational constant, $g=1 \mathrm{~m} / \mathrm{s}^{2}$, was employed. The computation was carried out using our algorithm with the WENO 3 scheme in the hyperbolic part, and the second-order finite difference method in the elliptic part. Non-reflecting boundary condition was used on the left and right boundaries during the computations. 

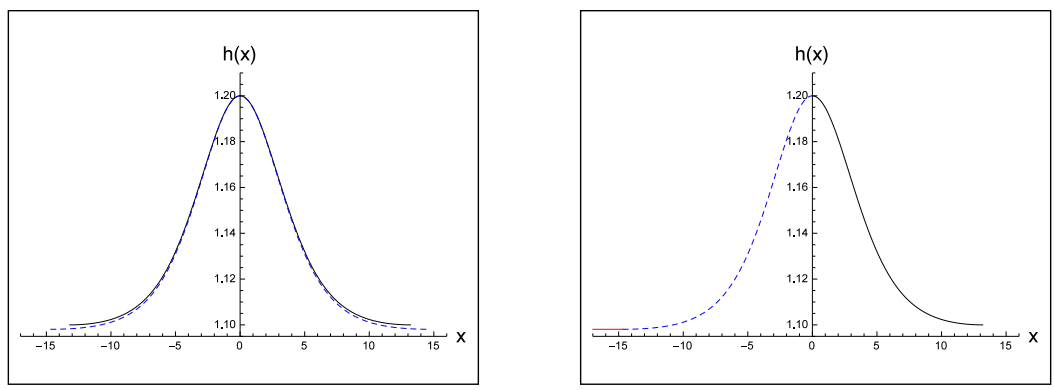

Figure 15: (a) One period of the function $h(x)$ is shown for the parameter choices $h_{0}, h_{1}, h_{2}$ (continuous line) and $h_{0}, h_{\star}, h_{2}$ (dashed line). (b) The initial smooth data for $h(x)$ joining the constant state 'star' (red line) and the initial wave train at the state $h_{2}$ shown by dashed line.

\section{Smoothing procedure}

Here we explain how we interpolated the initial discontinuity connecting the constant state $h \equiv h_{\text {* }}$ with the periodic wave train (with the period $L$ ). While the interpolation scale responsible for the smoothing out of the jump was a crucial parameter in [15], in our case this parameter was found to be irrelevant because independently of the initial conditions, the transition zone quickly acquires the 'half-solitary-wave-type' structure discussed in the main text.

Below, we present two types of interpolations which we used. The first type relies on the smoothing over the length scale $L_{\star} / 2$ where $L_{\star}$ is the length of the periodic wave with parameters $h_{0}, h_{\star}$ and $h_{2}$. More precisely, let the initial discontinuity be at $x=0$. We replace $h$ at the interval $\left[-L_{\star} / 2,0\right]$ by the explicit solution of $(35)$ with $h_{1}=h_{\star}=h_{\star}^{-}$:

$$
h(x)=h_{\star}+\left(h_{2}-h_{\star}\right) \mathrm{cn}^{2}\left(\kappa_{\star} x, s_{\star}\right), \quad x \in\left[-L_{\star} / 2,0\right],
$$

where $\kappa_{\star}^{2}=\frac{3}{4} \frac{h_{2}-h_{0}}{h_{0} h_{\star} h_{2}}$, and $s_{\star}^{2}=\frac{h_{2}-h_{\star}}{h_{2}-h_{0}}$. Such initially regularized curve (dashed curve in Figure 15 (left graph )) joins smoothly the constant solution $h=h_{\star}^{-}$at the point $x=-L_{\star} / 2$ with the periodic wave train at maximum $h=h_{2}$ (right graph in Figure 15). The expression for the velocity $u$ is obtained from the mass conservation condition $u(x)=m / h(x)$.

The second type of interpolation is optimal in the sense that it bridges the states $h_{\star}^{-}$and $h_{2}$ by a half of the solitary wave (see Figure 5) :

$$
h(x)=h_{\star}^{-}+\left(h_{2}-h_{\star}^{-}\right) \operatorname{sech}^{2}\left(\frac{x}{2} \sqrt{\frac{3\left(h_{2}-h_{\star}^{-}\right)}{h_{2}\left(h_{\star}^{-}\right)^{2}}}\right), \quad u(x)=\frac{m}{h(x)} .
$$

Both types of interpolation give the same final structure : the state 'star' is related with the wave train by a half of the solitary wave. In the main text we also show that even a drastic departure from the smooth interpolation approach, where we start with discontinuous initial data, still brings us to the same stationary profile of the shock-like transition front. 


\section{References}

[1] Bakholdin I 2004 Non-dissipative discontinuities in continuum mechanics, Fizmatlit, Moscow (in Russian)

[2] Batchelor C K 2000 An introduction to fluid dynamics. Cambridge University Press.

[3] Caflisch R E, Miksis M J, Papanicolaou G C and Ting L 1985 Effective equations for wave propagation in bubbly liquids. J. Fluid Mechanics 153 259-273.

[4] Clamond D, Dutykh D and Mitsotakis D 2017 Conservative modified Serre-Green-Naghdi equations with improved dispersion characteristics, Commun. Nonlinear Sci. Numer. Simulat. $45245-257$.

[5] Conforti M, Baronio F and Trillo S 2014 Resonant radiation shed by dispersive shock waves, Phys. Rev. A 89, 1013807.

[6] Courant R and Friedrichs K O 1999 Supersonic flow and shock waves. Vol. 21. Springer Science \& Business Media

[7] Dafermos C M 2000 Hyperbolic conservation laws in continuum physics. Vol. 325. Berlin: Springer

[8] Dias F and Milewski P 2010 On the fully-nonlinear shallow-water generalized Serre equations, Phys. Lett. A 374 1049-1053.

[9] DiPerna R J 1985 Measure-valued solutions to conservation laws, Archive for Rational Mechanics and Analysis 88, no. 3, 223-270.

[10] Di Pietro D A and Marche F 2018 Weighted interior penalty discretization of fully nonlinear and weakly dispersive free surface shallow water flows, J. Comput. Phys. 355 285-309.

[11] El G A, Geogjaev V V, Gurevich A V and Krylov A L 1995 Decay of an initial discontinuity in the defocusing NLS hydrodynamics, Physica D: Nonlinear Phenomena 87 186-192.

[12] El G A, Grimshaw R H J and Smyth N F 2006 Unsteady undular bores in fully nonlinear shallow-water theory, Phys. Fluids 18027104.

[13] El G A and Smyth N F 2016 Radiating dispersive shock waves in non-local optical media, Proc. R. Soc. A 472, no. 218720150633.

[14] El G A, Hoefer M A 2016 Dispersive shock waves and modulation theory, Physica D, 333 11-65.

[15] El G A, Hoefer M A and Shearer M 2016 Expansion shock waves in regularized shallow water theory, Proc. Royal Soc. A 47220160141.

[16] El G A, Hoefer M A and Shearer M 2017 Stationary expansion shocks for a regularized Boussinesq system, Studies Appl. Math. 140 27-47.

[17] Favrie N and Gavrilyuk S L 2017 A rapid numerical method for solving Serre-Green-Naghdi equations describing long free surface gravity waves, Nonlinearity 30 (7) 2718-2736. 
[18] Gavrilyuk S L 1994 Large amplitude oscillations and their 'thermodynamics' for continua with 'memory', European Journal of Mechanics B/ Fluids, 13 (6) 753-764.

[19] Gavrilyuk S L and Serre D 1995 A model of a plug-chain system near the thermodynamic critical point: connection with the Korteweg theory of capillarity and modulation equations, 419-428, in book : IUTAM Symposium on Waves in Liquid/Gas and Liquid/Vapor Two-Phase Systems, Eds. S. Morioka and L. van Wijngaarden, Kluwer Academic Publishers.

[20] Gavrilyuk S L and Teshukov V M 2001 Generalized vorticity for bubbly liquid and dispersive shallow water equations, Continuum Mechanics and Thermodynamics 13 365-382.

[21] Gavrilyuk S L, Gouin H and Teshukov V M 2004 Bubble effect on Kelvin - Helmholtz instability, Continuum Mech. Thermodyn. 16 31-42.

[22] Gelfand I M and Fomin S V 1991 Calculus of variations, Dover Publications, Mineola, New York.

[23] Green A E, Laws N and Naghdi P M 1974 On the theory of water waves, Proc. R. Soc. Lond. A $33843-55$.

[24] Green A E and Naghdi P M 1976 A derivation of equations for wave propagation in water of variable depth, J. Fluid Mech. 78 (1976), 237-246.

[25] Godlewski E and Raviart P A , Numerical Approximation of Hyperbolic Systems of Conservation Laws, Applied Mathematical Science 118, Springer-Verlag, 1996.

[26] Gurevich A and Pitaevskii L 1974 Nonstationary structure of a collisionless shock wave, JETP 38, 291 - 297.

[27] Gurevich A V and Krylov A L 1987 Dissipationless shock waves in media with positive dispersion, Zh. Eksp. Teor. Fiz. 92 1684-1699.

[28] Gottlieb S, Shu C W and Tadmor E 2001 Strong stability preserving high-order time discretization methods. SIAM Review, 43:89-112, 2001.

[29] Harten A, Lax P D and van Leer B 1983 On upstream differencing and Godunov-type schemes for hyperbolic conservation laws. SIAM Review 25 35-61.

[30] Hoefer M A and Ablowitz M J 2009 Dispersive shock waves, Scholarpedia 4 (11) 5562.

[31] Hoefer M A, Smith N F and Sprenger P 2019 Modulation theory solution for nonlinearly resonant, fifth-order Korteweg - de Vries, nonclassical, travelling dispersive shock waves, Stud. Appl. Math., 142 219-240.

[32] Hou T Y and Lax P D 1991 Dispersive approximations in fluid mechanics, Communications on Pure and Applied Mathematics, 44(1) (1991) 1-40.

[33] Kamchatnov A M 2000 Nonlinear Periodic Waves and Their Modulations: An Introductory Course, World Scinetific Publishing.

[34] Ketcheson D I and LeVeque R J 2008 WENOCLAW: A higher order wave propagation method, in: Hyperbolic Problems: Theory, Numerics, Applications, Springer-Verlag, 609-616. 
[35] Ketcheson D I, Parsani M and LeVeque R J 2013 High-order wave propagation algorithm for hyperbolic systems, SIAM J. Sci. Comput. 35(1) A351-A377.

[36] Lannes D 2013 The Water Waves Problem. Mathematical Surveys and Monographs, vol. 188 Amer. Math. Soc., Providence.

[37] Lax P D and Levermore C D 1983 The zero dispersion limit for the KdV equation, I-III." Comm. Pure Appl. Math. 36, 253-290.

[38] Lax P D 1991 The zero dispersion limit, a deterministic analogue of turbulence, Communications on Pure and Applied Math. 44, no. 89 1047-1056.

[39] Le Métayer O, Gavrilyuk S L and Hank S 2010 A numerical scheme for the Green-Naghdi model, J. Comp. Phys. 229 2034-2045.

[40] LeVeque, R J 2002 Finite Volume Methods for Hyperbolic Problems, Cambridge University Press.

[41] LeVeque R J 2007 Finite Difference Methods for Ordinary and Partial Differential Equations: Steady-State and Time-Dependent Problems, SIAM, Philadelphia.

[42] Li M, Guyenne P, Li F and Xu L 2014 High order well-balanced CDG-FE methods for shallow water waves by a Green-Naghdi model, J. Comp. Physics 257 169-192.

[43] Makarenko N 1986 A second long-wave approximation in the Cauchy-Poisson problem Dynamics Cont. Media 77 56-72 (in Russian).

[44] Maugin G A 1993 Material inhomogeneities in elasticity (Vol. 3). CRC Press.

[45] Miles J and Salmon R 1985 Weakly dispersive nonlinear gravity waves, J. Fluid Mechanics 157 $519-531$.

[46] Mindlin R D and Hermann G 1952 A one dimensional theory of compressive waves in elastic rod, In Proc. 1st US Nat. Congr. Appl. Mech.(ASME), 187-197.

[47] Mindlin R D 1965 Second gradient of strain and surface-tension in linear elasticity. International Journal of Solids and Structures, 1(4) 417-438.

[48] Pitti J P A, Zoppou C and Roberts S G 2017 Behaviour of the Serre equations in the presence of steep gradients revisited, arXiv:1706.08637v1 [math NA] 27 Jun 2017.

[49] Rayleigh J W S 1945 The theory of sound, V. 1. Dover Publications, New York .

[50] Saut J C and Xu L 2012 Well-posedness on large time for a modified full dispersion system of surface waves. J. Math. Phys. 53115606.

[51] Serre F 1953 Contribution à l'étude des écoulements permanents et variables dans les canaux, La Houille Blanche 8 (1953), 374-388.

[52] Serre D 1996 Systèmes de lois de conservation (I), Paris, Diderot, Editeur Arts Sciences.

[53] Shu C W 2009 High order weighted essentially nonoscillatory schemes for convection dominated problems, SIAM Review, 5 82-126. 
[54] Shyue K M and Xiao F 2014 An Eulerian interface sharpening algorithm for compressible two-phase flow: The algebraic THINC approach, J. Comput. Phys., 268 326-354.

[55] Smoller J 2012 Shock waves and reactiondiffusion equations 258 Springer Science \& Business Media.

[56] Sprenger P and Hoefer M A 2017 Shock waves in dispersive hydrodynamics with nonconvex dispersion, SIAM J. Appl. Math. 77 26-50.

[57] Sprenger P and Hoefer M A 2020 Discontinuous shock solutions of the Whitham modulation equations as dispersionless limits of travelling waves, Nonlinearity 33 3268-3302.

[58] Steinbach O 2007 Numerical Approximation Methods for Elliptic Boundary Value Problems: Finite and Boundary Elements, Springer.

[59] Su C H and Gardner C S 1969 Korteweg - de Vries Equation and Generalisations. III. Derivation of the Korteweg - de Vries Equation and Burgers Equation, J. Math. Physics, 10 536-539.

[60] Tkachenko S, Gavrilyuk S and Shyue K M 2019 Hyperbolicity of the modulation equations for the Serre-Green-Naghdi model, https://arxiv.org/abs/1904.07276

[61] Toro E F 1997 Riemann Solvers and Numerical Methods for Fluid Dynamics, Springer-Verlag, Berlin, Heidelberg.

[62] Truskinovsky L 1982 Equilibrium phase boundaries, Sov. Phys. Dokl. 27 551-553.

[63] Truskinovsky L 1993 Kinks versus shocks, In : Shock induced transitions and phase structures in general media, ed. J. E. Dunn, R. Fosdick, M. Slemrod, p. 185-229, Springer.

[64] Truskinovsky L and Zanzotto G 1996 Ericksen's bar revisited : energy wiggles, J. Mech. Phys. Solids, 44 1371-1408.

[65] Van der Waals J D 1893 The thermodynamic theory of capillarity under the hypothesis of a continuous density variation. J. Stat. Phys. 20 200-244 (translated by Rowlinson J S).

[66] Whitham G B 1974 Linear and Nonlinear Waves, John Wiley and Sons. 